\title{
An elastoplastic framework for granular materials becoming cohesive through mechanical densification. Part I - small strain formulation
}

\author{
Andrea Piccolroaz, Davide Bigoni and Alessandro Gajo \\ Dipartimento di Ingegneria Meccanica e \\ Strutturale, Università di Trento, \\ Via Mesiano 77, I-38050 Trento, Italia \\ email: piccolroaz@ing.unitn.it, bigoni@ing.unitn.it,gajo@ing.unitn.it
}

August 2, 2004

\begin{abstract}
Mechanical densification of granular bodies is a process in which a loose material becomes increasingly cohesive as the applied pressure increases. A constitutive description of this process faces the formidable problem that granular and dense materials have completely different mechanical behaviours (nonlinear elastic properties, yield limit, plastic flow and hardening laws), which must both be, in a sense, included in the formulation. A treatment of this problem is provided here, so that a new phenomenological, elastoplastic constitutive model is formulated, calibrated by experimental data, implemented and tested, that is capable of describing the transition between granular and fully dense states of a given material. The formulation involves a novel use of elastoplastic coupling to describe the dependence of cohesion and elastic properties on the plastic strain. The treatment falls within small strain theory, which is thought to be appropriate in several situations; however, a generalization of the model to large strain is provided in Part II of this paper.
\end{abstract}

Keywords: Elastoplasticity; Granular materials; Mechanical densification; Forming; Ceramic Materials. 


\section{Introduction}

\subsection{A premise and the central problem}

Cold powder compaction is a process in which granular materials are made cohesive through mechanical densification. Subsequent sintering usually completes the treatment and yields the desired mechanical properties of the final piece. Since this process permits an efficient production of parts ranging widely in size and shape to close tolerances (Reed, 1995), there is an evident related industrial interest. For instance, metallurgical (German, 1984), pharmaceutical (Lordi and Cuitiño, 1997) and forming of traditional (e.g. ceramic tiles and porcelain products) and structural (e.g. chip carriers, spark plugs, cutting tools) ceramics represent common applications.

A crucial step in the above technique is the production of green bodies (i.e. the solids obtained after cold forming of ceramic powders) possessing enough cohesion to remain intact after mold ejection, being essentially free of macro defects, and handleable without failure in the subsequent treatments. This is not an easy task and indeed defects of various types are always present in the greens (Deis and Lannutti, 1998; Ewsuk, 1997; Hausner and Kumar-Mal, 1982; Thompson, 1981b), negatively affecting local shrinkage during sintering. In particular, defects can be caused by the densification process, which may involve highly inhomogeneous strain fields, or by mold ejection].

In the present article a new elastoplastic phenomenological model is formulated, developed and implemented, which is capable of describing the deformation and consequent gain in cohesion of granular ceramic materials subject to mechanical loading. The model is calibrated by experimental data relative to a ready-to-press alumina powder, and numerical predictions are compared to experiments performed on simple forming processes. The proposed constitutive framework allows the possibility of simulating cold forming of pieces and to predict inhomogenities in cohesion, density, and elastic properties and residual stress distribution within the green, thus allowing a rational design of pieces.

Models aimed at achieving results similar to those addressed here have been developed for metal powders (Ariffin et al. 1998; Brown and Abou-Chedid, 1994; Brown and Weber, 1988; Khoei and Lewis, 1999; Lewis and Khoei, 1998; Lewis et al. 1993; Oliver et al. 1996; Redanz, 1999; 2001; Redanz and Tvergaard, 2003; Sun and Kim, 1997) and for ceramic granular materials (Ahzi et al. 1993; Aydin et al. 1997a,b; Brandt and Nilsson, 1998; 1999; Ewsuk et al. 2001; Keller et al. 1998; Piccolroaz et al. 2002; Zipse, 1997). Due to the plasticity of grains, metal powders are essentially different from ceramics, but nevertheless we point out that both the above classes of models cannot describe the features considered in the present paper ${ }^{2}$. In particular, the central point is that

\footnotetext{
${ }^{1}$ Glass and Ewsuk (1997) classify several types of damage such as for instance end and ring capping, laminations, shape distortions, surface defects, vertical cracks, and large pores.

${ }^{2}$ The model employed by Piccolroaz et al. (2002) is simply a modified Cam-clay calibrated on experimental results relative to ceramic powders. It is similar to many other available in the literature
} 
the process of mechanical compaction requires the description of the transition from a granular to a dense or even a fully dense state. Since granular materials are characterized by mechanical properties much different from those typical of dense solids, the constitutive modelling must describe a transition between two distinctly different states of a material.

The above constitutive description has been obtained for the first time in the present article by suggesting a novel use of elastoplastic coupling theory (Hueckel, 1976), introducing two micromechanically based hardening rules, and employing a recently proposed yield function (Bigoni and Piccolroaz, 2004).

It may be also important to mention that, even if the emphasis is placed here on materials where cohesion increases with pressure, 'vice-versa', we believe our constitutive framework to be sufficiently general to describe progressive decohesion due to mechanically-induced damage of quasibrittle materials (concrete, cemented sand, and rock), a problem investigated by Lagioia and Nova (1995).

Ceramic forming may involve deformations up to $50 \%$ and even greater, so that the need for a large strain formulation could be advocated. However, numerical simulations (some of which will be presented later) show that at least the 'gross' material behaviour is dominated by nonlinearities already occurring when deformations are small, so that the small strain formulation presented here should not be considered inappropriate. For completeness, nevertheless, a large strain formulation, requiring a new extension of the concept of elastoplastic coupling, is given in the Part II of this article (Piccolroaz et al. 2005).

\subsection{The densification of ceramic powders and the related diffi- culties in the constitutive modelling}

The compaction of a ceramic powder is a process essentially consisting of three phases: (I) granule sliding and rearrangement, (II) granule deformation, and (III) granule densification. For obvious reasons, a sharp differentiation between the three phases cannot be established, but qualitative morphologies of granular arrangements during Phases I and II are shown respectively in the central and lower parts of Fig. 1, with respect to the ceramic powder employed in our study (392 Martoxid KMS-96, defined in Appendix A and shown in the upper part of the figure in its loose state).

The gain in cohesion starts with Phase II, which is marked by the so called 'breakpoint pressure', conventionally denoted by a change in inclination of the semi-logarithmic plot of density versus applied pressure (Fig. 2, referred to ceramic tablets obtained by uniaxial pressing of 392 Martoxid KMS-96 alumina powder). Since the densification process is often highly inhomogeneous, usually at least two phases coexist. Phase I always occurs

and even if it can be considered perhaps sufficient for certain engineering purposes, it is far from realistic and does not address the major difficulties in modelling the densification process. 

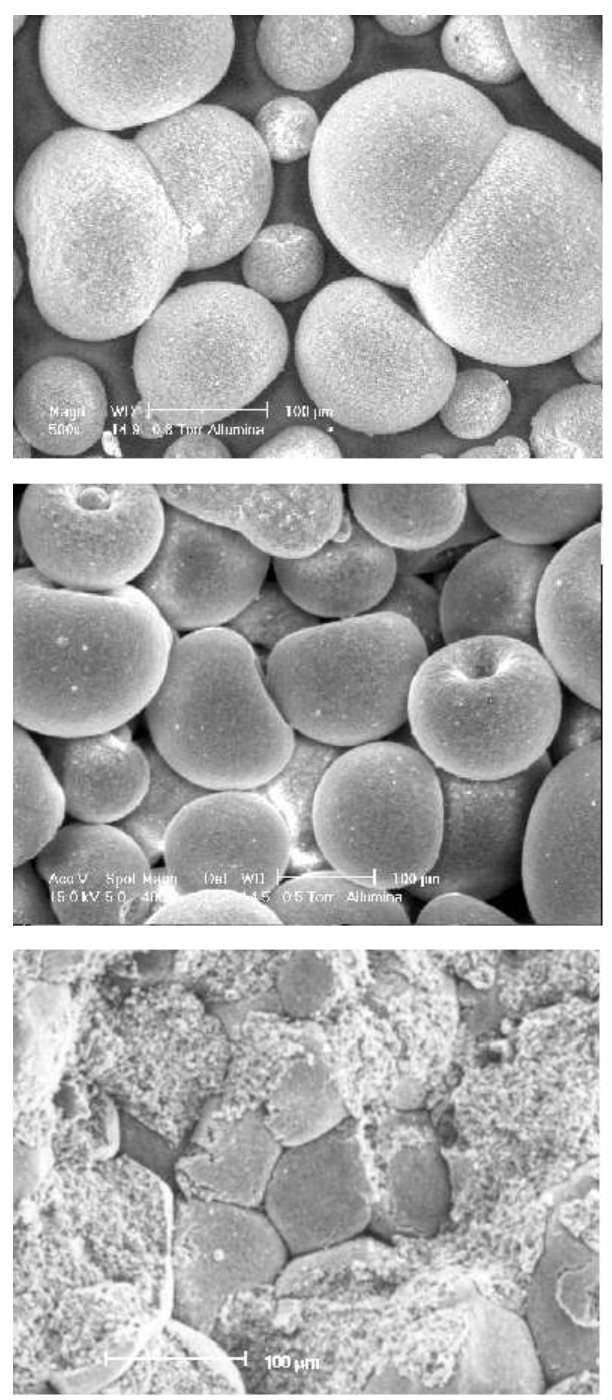

Figure 1: Micrographs of M KMS-96 alumina powder. The loose state is shown in the upper part, while granule arrangements corresponding to Phases I and II are shown in the central and lower part of the figure.

in early volumetric deformation of granular materials, so that it has been thoroughly investigated for geomaterials and, as will be explained later, is characterized by an elasticplastic behaviour in which the elastic response is nonlinearly dependent on mean stress. In contrast to Phase I, the other phases have been much less explored. The increase in cohesion 3 becomes already substantial during Phase II, as shown in Fig. 3, where

\footnotetext{
${ }^{3}$ The degree of induced cohesion during mechanical densification depends on the applied pressure and is also strongly influenced by several factors, including stiffness, ductility and shape of the particles
} 


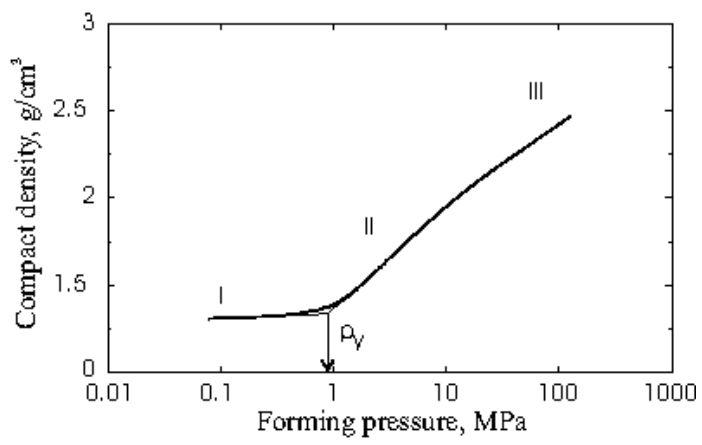

Figure 2: Compaction diagram of M KMS-96 alumina powder. The breakpoint pressure (expressed in terms of axial stress during uniaxial strain), separating Phase I from Phase II, is denoted by $p_{y}$. A few identical experiments have been performed, providing almost coincident curves, one of which is reported here.

cohesion measured (on the same tablets employed for Fig. 21) from biaxial flexure strength apparatus (defined by ASTM F 394) is reported versus the forming pressure (the axial compressive stress applied during uniaxial strain).

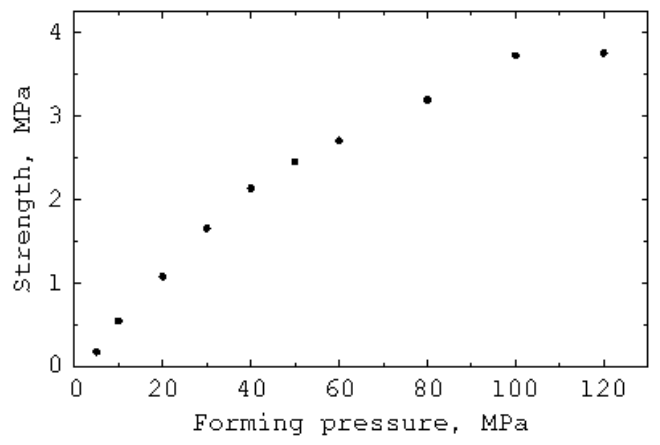

Figure 3: Biaxial flexure strength (following ASTM F 394) of green tablets from M KMS-96 alumina powder, as related to forming pressure under uniaxial strain in a cylindrical mold. Each point is the mean value taken over five tests, deviation was found to be negligible.

The peculiar mechanism of variation in cohesion due to plastic deformation can be described by making recourse to the concept of hardening. In particular, we will assume that a yield function exists for a granular material, defining its elastic range, so that when the material is in the initial cohesionless state, the null stress state lies on the yield

(Brown Abu Chedid, 1994). Experiments performed by D. Bigoni, G. Celotti, S. Guicciardi and A. Tampieri showed that penny-shaped particles produce a much higher increase in cohesion than sphericalshaped particles. 
surface, Fig. 4. Now, if the material is subject to increasing hydrostatic compression,

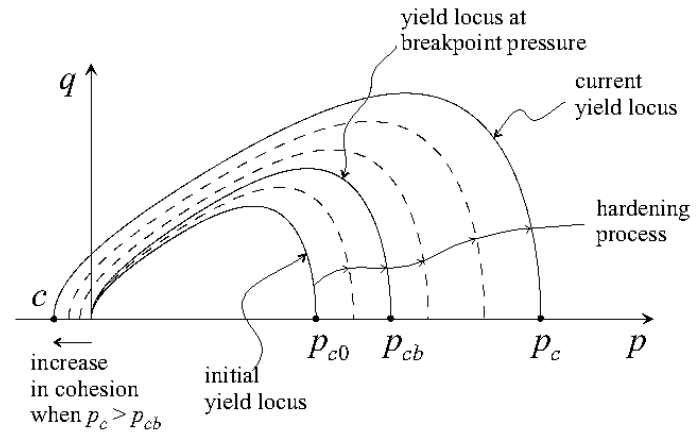

Figure 4: Increase in cohesion as related to hardening.

after an initial (small) deformation in the elastic range, an early development of plastic deformation occurs from a virgin state, corresponding to Phase I compaction. In this phase, the increase in cohesion is limited and almost negligible. However, when the pressure reaches the breakpoint value, so that material enters Phase II, the gain in cohesion becomes crucially important. In conclusion, to describe this process, we may employ a hardening law leading to a yield surface evolution of the type sketched in Fig. 4, where the yield surface shape distortion changes qualitatively, when the applied pressure $p$ exceeds the breakpoint pressure $p_{c b}$, expressed in terms of mean stress (with reversed sign) 4 .

At this level of description, one can get the impression that modelling the mechanism of cohesion increase during densification of granular materials could be pursued by simply employing an appropriate hardening rule. However, the elastic range of granular materials cannot be properly described by linear elasticity so that the elastic response of the material changes, when the elastic range is modified during hardening. More in detail, during Phase I of densification, studies relative to geomaterials demonstrate definitively that the elastic law relating the volumetric deformation to the applied mean pressure is logarithmic 5, as sketched in Fig. 5. Since the logarithmic law is simply not defined for a cohesionless material at null pressure, the increase in the cohesion of the material implies a modification to the elastic law, which becomes dependent on the plastic deformation, the physical quantity playing the role of the driving mechanism for densification. This

\footnotetext{
4 The type of increase in cohesion could also qualitatively change after the Phase III of densification is entered, but since we do not possess enough experimental data relative to this behaviour (occurring however at very high pressures, not involved in the usual forming of ceramics), this is not accounted for in the modelling. We believe anyway that its consideration would be not difficult, once experimental results were made available.

${ }^{5}$ This law has reached the status of unchallenged law for fine grained soils, whereas it is strictly followed only during elastic unloading in coarse grained materials (Lambe and Whitman, 1969).
} 
means that the elastic properties of the material must depend on the plastic deformation, a feature that can be described by making recourse to the concept of elastoplastic coupling (Hueckel, 1976; Dougill, 1976).

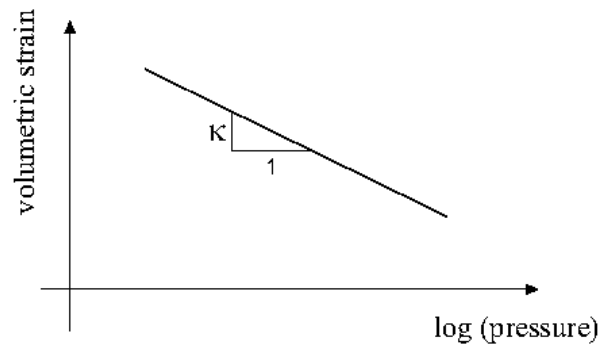

Figure 5: Logarithmic elastic law relating the elastic bulk modulus $\kappa$ to pressure (mean stress taken positive when compressive).

In the following, we will introduce the three fundamental ingredients in the modelling of the densification processes, namely, (i) the yield function appropriate for the description of the behaviour of granular materials, (ii) the nonlinear elastic model, coupled to plasticity, (iii) two micromechanically-based hardening laws.

\section{$1.3 \quad$ Summary}

Part I of this paper is organized as follows. After a brief explanation of notation, the constitutive laws are introduced in Sect. 2, together with the calibration to experimental results. Numerical simulations and comparisons to experimental results relative to simple forming processed are presented in Sect. 3, Extension of the constitutive model to large strain is deferred to Part II of this paper (Piccolroaz et al. 2005).

\subsection{Notation}

A standard, intrinsic notation is used throughout the paper, where vectors and secondorder tensors are denoted by bold (the latter capital) letters. The scalar product, the trace operator and the transpose are denoted by the usual symbols, namely,

$$
\boldsymbol{A} \cdot \boldsymbol{B}=\operatorname{tr} \boldsymbol{A} \boldsymbol{B}^{T}
$$

for every tensors $\boldsymbol{A}$ and $\boldsymbol{B}$. The following invariants of stress $\boldsymbol{\sigma}$ will be used

$$
p=-\frac{\operatorname{tr} \boldsymbol{\sigma}}{3}, \quad q=\sqrt{3 J_{2}}, \quad \theta=\frac{1}{3} \cos ^{-1}\left(\frac{3 \sqrt{3}}{2} \frac{J_{3}}{J_{2}^{3 / 2}}\right),
$$


where $\theta \in[0, \pi / 3]$ is the Lode's invariant and

$$
J_{2}=\frac{1}{2} \operatorname{dev} \boldsymbol{\sigma} \cdot \operatorname{dev} \boldsymbol{\sigma}, \quad J_{3}=\frac{1}{3} \operatorname{tr}(\operatorname{dev} \boldsymbol{\sigma})^{3}, \quad \operatorname{dev} \boldsymbol{\sigma}=\boldsymbol{\sigma}-\frac{\operatorname{tr} \boldsymbol{\sigma}}{3} \boldsymbol{I},
$$

in which $\operatorname{dev} \boldsymbol{\sigma}$ is the deviatoric stress and $\boldsymbol{I}$ is the identity tensor.

We will employ two tensorial products between second-order tensors $\boldsymbol{A}$ and $\boldsymbol{B}$, namely,

$$
(\boldsymbol{A} \otimes \boldsymbol{B})[\boldsymbol{C}]=(\boldsymbol{B} \cdot \boldsymbol{C}) \boldsymbol{A}, \quad(\boldsymbol{A} \underline{\otimes} \boldsymbol{B})[\boldsymbol{C}]=\boldsymbol{A} \frac{\boldsymbol{C}+\boldsymbol{C}^{T}}{2} \boldsymbol{B}^{T},
$$

so that $\boldsymbol{I} \bar{\otimes} \boldsymbol{I}$ becomes the symmetrizing fourth-order tensor, defined for every tensor $\boldsymbol{A}$ as $\boldsymbol{I} \underline{\bar{\otimes}} \boldsymbol{I}[\overline{\boldsymbol{A}}]=\left(\boldsymbol{A}+\boldsymbol{A}^{T}\right) / 2$.

\section{The constitutive model}

\subsection{The yield function}

During cold mechanical compaction of ceramic powders, the shape of the yield surface evolves from that typical of granular material (for instance, that of the modified CamClay model) to that characteristic of a ductile, dense material (for instance, that of the Gurson model). To describe this process in terms of hardening, a suitably 'deformable' surface is needed. This was found by Bigoni and Piccolroaz (2004); in particular, the yield function takes the form

$$
F\left(\boldsymbol{\sigma}, p_{c}, c\right)=f\left(p, p_{c}, c\right)+\frac{q}{g(\theta)}
$$

where $p_{c}$ and $c$ are the parameters that will be assumed to depend on plastic deformation and thus they will define the hardening behaviour, $q$ is the deviatoric invariant $(2)_{2}, f(p)$ is the function describing the dependence on the mean pressure $p$, eqn. (2) 1 , assumed in the form

$$
f\left(p, p_{c}, c\right)= \begin{cases}-M p_{c} \sqrt{\left(\Phi-\Phi^{m}\right)[2(1-\alpha) \Phi+\alpha]} & \text { if } \Phi \in[0,1] \\ +\infty & \text { if } \Phi \notin[0,1]\end{cases}
$$

in which

$$
\Phi=\frac{p+c}{p_{c}+c}
$$

and $g(\theta)$ describes dependence on the Lode's invariant $\theta$ defined by eqn. (2) ${ }_{3}$ taken as

$$
g(\theta)=\frac{1}{\cos \left[\beta \frac{\pi}{6}-\frac{1}{3} \cos ^{-1}(\gamma \cos 3 \theta)\right]} .
$$


The yield function described by eqn. (5)-(8) has been motivated and explained in great detail by Bigoni and Piccolroaz (2004), where the interested reader is remanded for details. We mention here that the yield surface corresponding to eqns. (515)-(8) is extremely versatile and remains convex in a broad range of values of parameters $M, p_{c}$, $c, m, \alpha, \beta, \gamma$.

The yield function gradient $\boldsymbol{Q}=\partial f / \partial \boldsymbol{\sigma}$, needed for the practical implementation of the model, is reported for completeness in Appendix B.

\subsubsection{Calibration of the parameter describing the yield function for alumina powder}

The problem now is to determine the yield surface parameters needed to describe the material considered here. For this purpose, we note that $m$ and $\alpha$, defining the shape of the meridian section, can be calibrated employing the experimental iso-density data in the $(p, q)$ plane obtained from triaxial compression tests by Bonnefoy (as reported by Kim et al. 2002), under the assumption that iso-density curves correspond to yield surface sections (Kim et al. 2000; Kim et al. 2002). The values $m=2$ and $\alpha=0.1$ were found to provide the best fitting, as shown in Fig. 6, where the data sets correspond to different levels of densification.

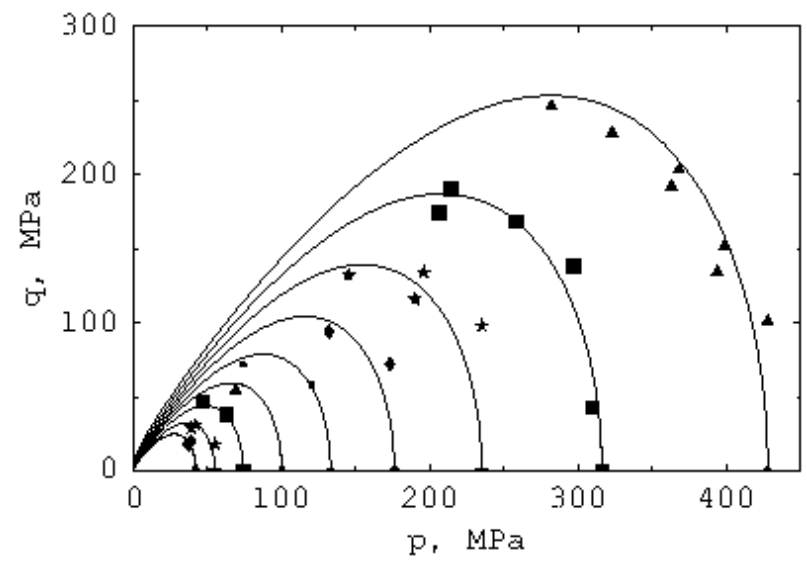

Figure 6: Meridian sections of the employed yield surface fitted to the iso-density data for alumina powder (taken from Kim et al. 2002) at different levels of densification (increase in cohesion is not appreciable at the scale of the figure).

To our knowledge, experimental data are not available to define the deviatoric section of the yield surface for alumina powder (and more in general for ceramic powders). Therefore, parameters $\beta$ and $\gamma$ have been calibrated on the basis of the value of the angle of internal friction, determined for our alumina powder from a standard geotechnical 
direct shear test apparatu 6 as follows.

The variation of the vertical displacement of the sample upper surface [a positive (negative) sign denotes a dilatant (contractant) behaviour] and of the applied shear force during shearing is shown in Fig. 7.
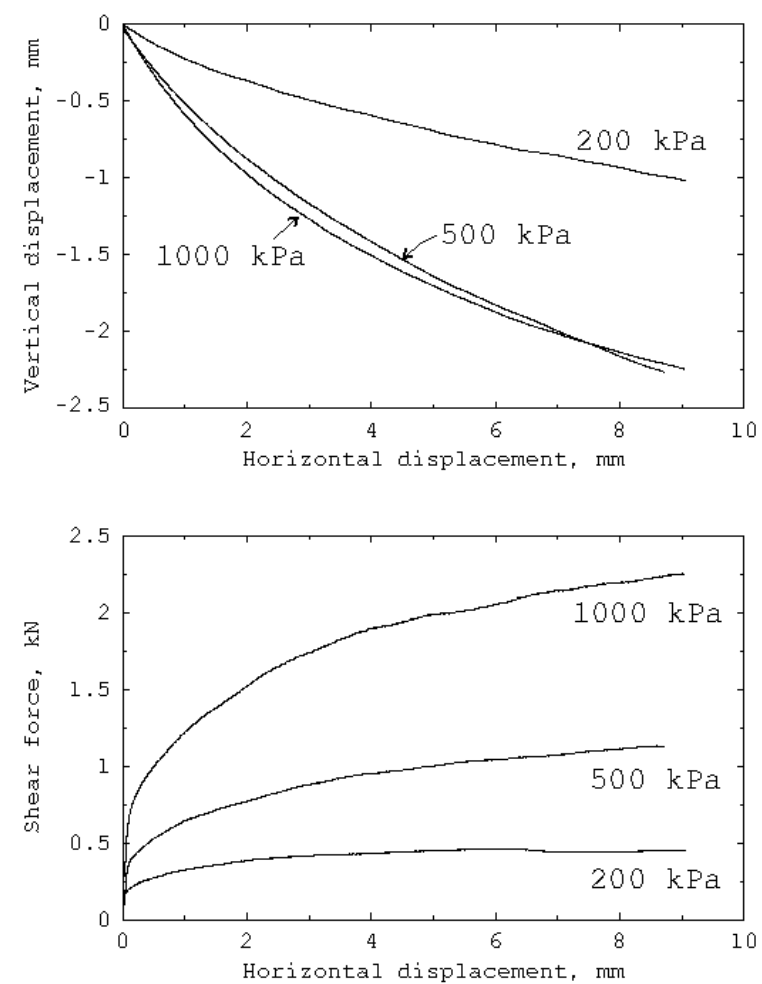

Figure 7: Vertical (negative sign corresponds to contractant behaviour) vs. horizontal displacements (upper part) and shear force vs. horizontal displacement (lower part) of three samples of M KMS-96 alumina powder, for different vertical pressures (200, 500 and $1000 \mathrm{kPa})$.

It can be observed from the figure that the tested samples exhibited the typical behaviour of a loose sand, with compressive volumetric strains during shearing, without a softening phase. The fact that the samples sheared at $500 \mathrm{kPa}$ and $1000 \mathrm{kPa}$ of vertical pressure have the same volumetric strains is probably related to a slightly looser initial

\footnotetext{
${ }^{6}$ The apparatus consists of a shear box containing the sample, which is split in the middle height. When a normal force is applied, the horizontal force required to induce a movement of the upper half of the sample with respect to the lower half is measured. This test is useful for the evaluation of the friction angle of a granular material, like the alumina powder in Phase I of densification. The samples were formed by carefully pouring the ceramic powder within the shear box. Shearing was performed at a velocity of $0.2 \mathrm{~mm} / \mathrm{min}$.
} 
condition of the former sample. It can be observed that, except for the test performed under a vertical stress of $200 \mathrm{kPa}$, the steady state condition typical of the critical state is not reached and at the end of the test the strength and the volumetric strains of the samples are still slightly increasing. This effect is more pronounced at larger applied vertical pressures and is probably connected to the progressive deformation and rupture of the grains constituting the alumina powder occurring during shearing even at low confining pressures. This is consistent with the experimental evidences that very large shear strains are necessary to reach the steady state in sands, when grain crushing occurs.

The maximum shear force reached at the end of the tests is plotted in Fig. 8 as a function of the applied vertical load. The results clearly lie on a straight line and may be interpreted following the Coulomb-Mohr failure criterion, to yield a friction angle approximately equal to $32^{\circ}$. Making recourse now to the Coulomb-Mohr model, the

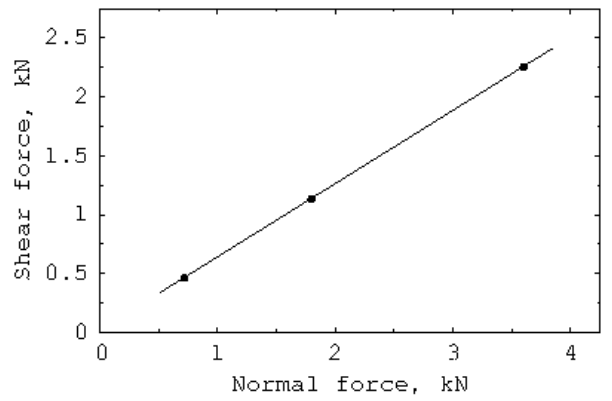

Figure 8: Direct shear test on M KMS-96 alumina powder.

ratio of the deviatoric section radius for triaxial extension, $g(0)$, to that for triaxial compression, $g(\pi / 3)$, is related to the angle of internal friction through

$$
\frac{g(0)}{g(\pi / 3)}=\frac{3-\sin \phi}{3+\sin \phi}
$$

Eqn. (9) is not enough to determine the two unknowns $\beta$ and $\gamma$. In the absence of further indications, parameter $\gamma$ was fixed to be equal to 0.9 , providing a deviatoric section fairly close to the piecewise linear deviatoric section (corresponding to $\gamma=1$ ). From this value and employing eqn. (9),$\beta=0.19$ follows.

Parameter $M$, defining the pressure-sensitivity, has been calibrated making recourse to the concept of critical state, a peculiar state in which a granular material deforms at constant stress and constant volume. Since the critical state occurs at null plastic (and irreversible) volumetric strain rates, which (for the flow rule that will be specified later) is equivalent to $\operatorname{tr} \boldsymbol{Q}=0$, the expression for the yield function gradient eqn. (B.1)-(B.3) provides the following condition

$$
2(m+1)(1-\alpha) \Phi^{m}+m \alpha \Phi^{m-1}-4(1-\alpha) \Phi-\alpha=0,
$$


which identifies the critical state. This implicit relation can be solved numerically, as soon as values for $m$ and $\alpha$ have been selected, providing the value of $\Phi$ corresponding to the critical state,

$$
\Phi^{\star}=\Phi^{\star}(m, \alpha) .
$$

In our case $\Phi^{\star}=0.658$ is obtained. The critical state point in the $(p, q)$ plane is therefore

$$
\left\{\begin{array}{l}
p^{\star}=\left(p_{c}+c\right) \Phi^{\star}-c, \\
q^{\star}=g(\theta) M p_{c} \sqrt{\left(\Phi^{\star}-\Phi^{\star m}\right)\left[2(1-\alpha) \Phi^{\star}+\alpha\right]},
\end{array}\right.
$$

Eqns. (11) define, through parameter $p_{c}$, the representation of the critical state line in the $(p, q)$ plane. Due to the fact that $c$ is a nonlinear function of $p_{c}$, the critical state line results mildly curved 7 . Indeed, the line is straight only in the first phase of densification, as long as $c=0$, and then deflects from linearity in the subsequent phase, when cohesion increases, approaching, after substantial plastic deformation and $c \sim c_{\infty}$, again a straight line with the same slope as the initial line. In other words, the final inclination of the critical state line, reached after substantial increase in cohesion, is

$$
\frac{d q}{d p}=g(\theta) M \frac{\sqrt{\left(\Phi^{\star}-\Phi^{\star m}\right)\left[2(1-\alpha) \Phi^{\star}+\alpha\right]}}{\Phi^{\star}} .
$$

It can be observed from eqn. (11) that, since $c<<p_{c}$ throughout the densification process, the effect of $c$ can be neglected, so that eqn. (12) can be taken as the slope of the critical state line for the entire deformation process. With reference to the triaxial compression state, $\theta=\pi / 3$, this slope is related to the angle of internal friction through

$$
\frac{d q}{d p}=\frac{6 \sin \phi}{3-\sin \phi}
$$

which gives a value of $M$ equal to 1.1 .

\section{$2.2 \quad$ Elastoplastic coupling}

Dependence of elastic response of a material on plastic deformation for describing degradation of elastic properties was suggested independently by Hueckel (1976) for soils and Dougill (1976) for concrete. The model was later developed by Hueckel and Maier (1977), Capurso (1979), Maier and Hueckel (1979), and Bigoni and Hueckel (1991). We will develop the concept of elastoplastic coupling in the way suggested by Bigoni (2000), which yields a symmetric constitutive operator in the specific case of associative flow rule. This fact follows from different constitutive assumptions, in particular, it will be assumed here

\footnotetext{
7 Note that the definition of critical state is extended here to include cohesive and ductile materials; we assume for simplicity that cohesion does not change at critical state.
} 
as in (Bigoni, 2000; Gajo et al. 2004) that the flow rule sets the so-called 'irreversible' strain rate, which in the case of coupling is different from the plastic strain rate.

The necessity of elastoplastic coupling for modelling the densification process of granular materials considered here may be motivated — as sketched in Fig. 9 - by the observation that elastic unloading in a uniaxial deformation test shows a clear tendency toward a stiffening caused by the increase in cohesion. However, even if this effect would

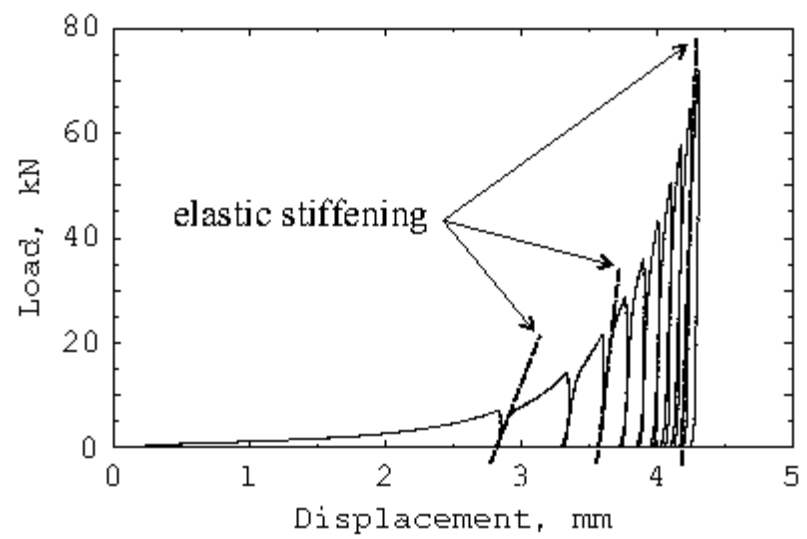

Figure 9: Elastic stiffening during uniaxial deformation test (experimental results on M KMS-96 alumina powder).

be disregarded in a first approximation, elastoplastic coupling would always be needed, for the reason mentioned in the Introduction, namely, to match the increase in cohesion with the nonlinear, logarithmic elastic model usually accepted for granular materials.

The basic concept of the elastoplastic coupling is that the elastic potential $\phi$ depends, in addition to the elastic, also on the plastic strain, so that

$$
\boldsymbol{\sigma}=\frac{\partial \phi\left(\boldsymbol{\epsilon}^{e}, \boldsymbol{\epsilon}^{p}\right)}{\partial \boldsymbol{\epsilon}^{e}},
$$

where $\boldsymbol{\epsilon}^{e}$ and $\boldsymbol{\epsilon}^{p}$ are the elastic and plastic components of deformation, respectively (obeying the usual addittive rule).

\subsection{Elastic potential for compaction during Phase I and early Phase II}

In the elastic range, granular cohesionless material obey the well-known logarithmic law sketched in Fig. 5, relating the increment in the void ratio $\Delta e=e-e_{0}$ (measured with respect to an initial value $e_{0}$ ) to the current mean pressure

$$
\Delta e^{e}=-\kappa \log \frac{p}{p_{0}},
$$


where the suffix $e$ remarks that we are referring to the elastic range, $p_{0}$ is the value of $p$ corresponding to the initial void ratio $e_{0}$, and $\kappa$ is the logarithmic bulk modulus. Eqn. (15) is the starting point to obtain the nonlinear elastic potential employed in the Cam-clay model (Roscoe and Schofield, 1963; Roscoe and Burland, 1968), which is particularly suitable for the description of cohesionless granular media, or, in other word, during Phase I of compaction]. However, our intention here is to describe the behaviour of materials which may increase (or decrease) cohesion as a function of the plastic deformation. Therefore, we have to introduce a modification in the elastic Camclay potential, first, to include a cohesion and, second, to make this dependent on plastic deformation. The easiest way to do this is to introduce a plastic-dependent cohesion in eqn. (15), playing the role of a modification to the mean pressure

$$
\Delta e^{e}=-\kappa \log \frac{p+c\left(\boldsymbol{\epsilon}^{p}\right)}{p_{0}+c\left(\boldsymbol{\epsilon}^{p}\right)}
$$

where $c\left(\boldsymbol{\epsilon}^{p}\right)$ is the (positive) parameter describing the cohesion and depending on plastic deformation. In particular, the cohesion is assumed to depend on the volumetric component only of plastic deformation. This may be motivated by qualitative micromechanical considerations. Following, for instance, the Rowe (1962) model of a granular material, a shear deformation yields a decrease (increase) of cohesion when accompanied by dilatancy (contractivity), Fig. 10, Obviously, the relation (16) is meaningful only in an early

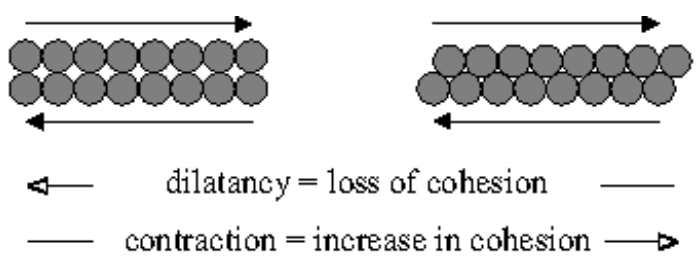

Figure 10: The mechanism of increase and loss of cohesion visualized in terms of the Rowe model.

stage of Phase II compaction, since unrealistic small values of void ratio are predicted at increasing $p$. However, it is important to realize that the description of the initiation of Phase II is crucial, since the material goes from a cohesionless state to a solid state.

8 The value of the logarithmic bulk modulus $\kappa$, which governs the elastic behaviour of the material in the first phase of densification, was deduced from experimental results for the alumina powder considered here by measuring the slope of the curves obtained by loading and unloading in uniaxial strain tests. For this evaluation, we have assumed a constant ratio between the horizontal $\sigma_{h}$ and vertical $\sigma_{v}$ stresses equal to 0.47 , as deduced from the formulae

$$
\frac{\sigma_{h}}{\sigma_{v}}=1-\sin \phi,
$$

which is usually employed for granular media (Jaky, 1944). 
We are in a position now to proceed with eqn. (16) in the way usually followed in the derivation of the Cam-clay model, i.e. in the case of eqn. (15). Therefore, assuming incompressibility of grains, the volumetric elastic deformation is given by $\left(e-e_{0}\right) /\left(1+e_{0}\right)$, so that eqn. (16) defines a volumetric nonlinear elastic law, to be added to a linear elastic deviatoric constitutive equation. The resulting elastic potential is therefore

$$
\phi\left(\boldsymbol{\epsilon}^{e}, \boldsymbol{\epsilon}^{p}\right)=-\frac{\mu}{3}\left(\operatorname{tr} \boldsymbol{\epsilon}^{e}\right)^{2}+c \operatorname{tr} \boldsymbol{\epsilon}^{e}+\tilde{\kappa}\left(p_{0}+c\right) \exp \left(-\frac{\operatorname{tr} \boldsymbol{\epsilon}^{e}}{\tilde{\kappa}}\right)+\mu \boldsymbol{\epsilon}^{e} \cdot \boldsymbol{\epsilon}^{e},
$$

where $\mu$ is the elastic shear modulus and $\tilde{\kappa}=\kappa /\left(1+e_{0}\right)$.

The elastic potential (17), with parameters $\mu$ and $\kappa$ taken independent of the plastic deformation, is certainly suitable to describe the behaviour of the material in Phase I of the compaction process, since here the material is still granular and $c=0$, so that the model reduces to the celebrated Cam-clay model. Moreover, the elastic potential (17) can describe the initiation of Phase II of densification, where the increase in cohesion starts to play a role. On the other hand, the potential (17) embodies a nonlinear increase of elastic bulk modulus with mean pressure, a feature which is clearly unrealistic during the late Phase II of deformation.

\subsection{Elastic potential for compaction during the late Phase II}

In the late Phase II, the material becomes more similar to a porous solid than to a granular body so that its properties progressively change. Experimental evidence of the mechanical behaviour of green's bodies formed at pressures corresponding to Phase II deformation is scarce. In particular, experiments by Zeuch et al. (2001) on alumina powder evidence that elastic properties become linear functions of forming pressure $p_{c}$. Therefore, an elastic potential such as

$$
\phi\left(\boldsymbol{\epsilon}^{e}, \boldsymbol{\epsilon}^{p}\right)=\left(\frac{K}{2}-\frac{\mu}{3}\right)\left(\operatorname{tr} \boldsymbol{\epsilon}^{e}\right)^{2}+\mu \boldsymbol{\epsilon}^{e} \cdot \boldsymbol{\epsilon}^{e}
$$

can be expected, where both $\mu$ and $K$ are linear functions of $p_{c}$. Though eqn. (18) is very simple, we believe that the introduction of more complicated laws would be straightforward.

\subsection{Elastic potential for compaction during Phases I and II}

During deformation, the material behaviour changes from that described by potential (17) to that corresponding to potential (18). In order to describe this transition in the material behaviour, we modify the elastic potential (17) as follows

$$
\begin{aligned}
& \phi\left(\boldsymbol{\epsilon}^{e}, \boldsymbol{\epsilon}^{p}\right)=-\frac{\mu(d)}{3}\left(\operatorname{tr} \boldsymbol{\epsilon}^{e}\right)^{2}+c \operatorname{tr} \boldsymbol{\epsilon}^{e} \\
&+\left(p_{0}+c\right)\left[\left(d-\frac{1}{d}\right) \frac{\left(\operatorname{tr} \boldsymbol{\epsilon}^{e}\right)^{2}}{2 \tilde{\kappa}}+d^{1 / n} \tilde{\kappa} \exp \left(-\frac{\operatorname{tr} \boldsymbol{\epsilon}^{e}}{d^{1 / n} \tilde{\kappa}}\right)\right]+\mu(d) \boldsymbol{\epsilon}^{e} \cdot \boldsymbol{\epsilon}^{e},
\end{aligned}
$$


where $d \geq 1$ is a 'transition' parameter depending on the plastic volumetric strain through the forming pressure and governing the passage from logarithmic to linear law of elastic bulk modulus. Note that for $d=1$ and in the limit $d \longrightarrow \infty$ the potentials (17) and (18) are respectively recovered. Finally, $n \geq 1$ is a material constant defining the decay of the exponential term. Moreover, the elastic shear modulus $\mu$ is taken dependent on plastic volumetric strain through parameters $d$ and $c$ as follows

$$
\mu(d)=\mu_{0}+c\left(d-\frac{1}{d}\right) \mu_{1}
$$

where $\mu_{0}$, and $\mu_{1}$ are positive material constants.

In conclusion, the nonlinear elastic stress/strain law may be obtained from eqn. (19) and results dependent on the plastic strain through $c$ and $d$ (the dependence is often omitted in the following for conciseness)

$$
\begin{aligned}
\boldsymbol{\sigma}=\left\{-\frac{2}{3}\right. & \mu \operatorname{tr} \boldsymbol{\epsilon}^{e}+c \\
& \left.+\left(p_{0}+c\right)\left[\left(d-\frac{1}{d}\right) \frac{\operatorname{tr} \boldsymbol{\epsilon}^{e}}{\tilde{\kappa}}-\exp \left(-\frac{\operatorname{tr} \boldsymbol{\epsilon}^{e}}{d^{1 / n} \tilde{\kappa}}\right)\right]\right\} \boldsymbol{I}+2 \mu \boldsymbol{\epsilon}^{e} .
\end{aligned}
$$

Taking the time derivative 9 of (21), we get the rate equations

$$
\begin{gathered}
\dot{\boldsymbol{\sigma}}=\mathbb{E}\left[\dot{\boldsymbol{\epsilon}}^{e}\right]+\dot{c}\left[1+\left(d-\frac{1}{d}\right) \frac{\operatorname{tr} \boldsymbol{\epsilon}^{e}}{\tilde{\kappa}}-\exp \left(-\frac{\operatorname{tr} \boldsymbol{\epsilon}^{e}}{d^{1 / n} \tilde{\kappa}}\right)\right] \boldsymbol{I} \\
+\dot{d} \frac{p_{0}+c}{\tilde{\kappa}} \operatorname{tr} \boldsymbol{\epsilon}^{e}\left[1+\frac{1}{d^{2}}-\frac{1}{n d^{1+1 / n}} \exp \left(-\frac{\operatorname{tr} \boldsymbol{\epsilon}^{e}}{d^{1 / n} \tilde{\kappa}}\right)\right] \boldsymbol{I} \\
+\dot{\mu}\left(-\frac{2}{3} \operatorname{tr} \boldsymbol{\epsilon}^{e} \boldsymbol{I}+2 \boldsymbol{\epsilon}^{e}\right),
\end{gathered}
$$

where $\dot{c}, \dot{d}$, and $\dot{\mu}$ arise from elastoplastic coupling $(\dot{c}=\dot{d}=\dot{\mu}=0$ in the usual uncoupled models) and the elastic fourth-order tensor $\mathbb{E}$, together with its inverse $\mathbb{E}^{-1}$ (restricted to the space of all symmetric tensors) are given by

$$
\mathbb{E}=\left[-\frac{2}{3} \mu+K_{t}\right] \boldsymbol{I} \otimes \boldsymbol{I}+2 \mu \boldsymbol{I} \underline{\bar{\otimes}} \boldsymbol{I}, \quad \mathbb{E}^{-1}=\frac{2 \mu-3 K_{t}}{18 \mu K_{t}} \boldsymbol{I} \otimes \boldsymbol{I}+\frac{1}{2 \mu} \boldsymbol{I} \underline{\otimes} \boldsymbol{I},
$$

in which the tangent bulk modulus $K_{t}$ depends on the plastic deformation through $c$ and $d$ and on the elastic deformation in the following way

$$
K_{t}=\frac{p_{0}+c}{\tilde{\kappa}}\left[d-\frac{1}{d}+d^{-1 / n} \exp \left(-\frac{\operatorname{tr} \boldsymbol{\epsilon}^{e}}{d^{1 / n} \tilde{\kappa}}\right)\right] .
$$

\footnotetext{
${ }^{9}$ Time is intended here as a loading parameter, while the material behaviour is assumed inviscid.
} 
We need now to specify the particular dependence of the transition parameter $d$ on the forming pressure $p_{c}$. To this purpose, we note that, assuming the existence of a saturation threshold $c_{\infty}$ for the value of the cohesion, the asymptotic behaviours of the bulk modulus $K_{t}$, eqn. (24), and the shear modulus $\mu$, eqn. (20), as $d \rightarrow \infty$ are

$$
K_{t} \sim \frac{p_{0}+c_{\infty}}{\tilde{\kappa}} d \text { and } \mu \sim c_{\infty} \mu_{1} d
$$

respectively. Since experimental results by Zeuch et al. (2001) suggest that $\mu$ and $K_{t}$ become linear functions of $p_{c}$ for large values of forming pressure, inspired by (25), we assume for simplicity that parameter $d$ is a linear function of forming pressure $p_{c}$, for values of pressure superior to the breakpoint threshold $p_{c b}$, so that

$$
d=1+B<p_{c}-p_{c b}>
$$

where $B$ is a positive material parameter and the symbol $<>$ denotes the Macaulay brackets operator (defined for every scalar $\alpha$ as $\langle\alpha\rangle=(\alpha+|\alpha|) / 2$ ).

It has to be noted that the determination of parameters $B, \mu_{1}$ and $n$ is not easy. In principle, parameters $B$ and $\mu_{1}$ can be obtained matching the asymptotic behaviour (25) with experiments of the type performed by Zeuch et al. (2001). However, precise determination of elastic constants of green's bodies is certainly difficult, moreover, $n$ needs also to be determined. We have estimated $B, \mu_{1}$ on the basis of the experiments by Zeuch et al. (2001), finding the values $B=0.18 \mathrm{MPa}^{-1}, \mu_{1}=64$.

Regarding the constant $n$, we have plotted the evolution of the tangent elastic bulk modulus during a hypothetical isotropic compression test at different values of $n$. This has been possible employing eqn. (24) with $d$ given by eqn. (26) and formula (32) for the cohesion $c$ that will be introduced later. In this way, $K_{t}$ depends on the forming pressure $p_{c}$ (which has been taken coincident with $-\operatorname{tr} \boldsymbol{\sigma} / 3$ ) and on $\operatorname{tr} \boldsymbol{\epsilon}$, which has been evaluated numerically solving the trace of eqn. (21). Results have been reported in Fig. 11 for $n=$ $\{1,6,60,600\}$, from which we note that for $n=1$ unphysical behaviours appear, but these are not any more evident starting from $n=6$. In the absence of experimental results, we have taken $n=6$ in our analyses. We note also that sensitivity to this parameter becomes very low for values of $n$ superior to 60 , so that a refined determination of parameter $n$ would require ad hoc experimental investigation.

\subsection{Two micromechanically-based hardening laws}

In order to further develop eqn. (22), evolution laws for the hardening parameters representing the forming pressure $p_{c}$ and the cohesion $c$ are needed, providing the functional dependences of these parameters on the plastic deformation. At this point, recourse to micromechanical considerations and experimental evidence becomes necessary. 


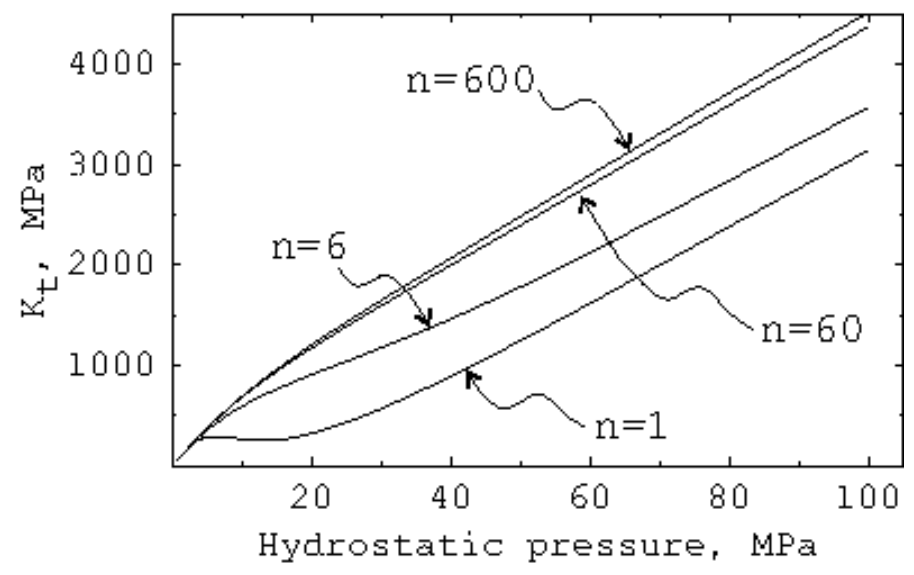

Figure 11: Simulated evolution of tangent elastic bulk modulus during isotropic compression. Four values of $n$ are considered: $n=1,6,60,600$.

\subsubsection{The relation between the forming pressure $p_{c}$ and plastic volumetric strain}

Parameter $p_{c}$ is related to the plastic deformation and this relationship can be determined employing the micromechanical model proposed by Cooper and Eaton (1962). This takes into account the fundamental fact that compaction can be divided into the three phases mentioned in the introduction. Based on statistical micromechanics considerations and validated on several experimental results on ceramic powders, Cooper and Eaton (1962) provide a double-exponential law describing the first two phases of densification in terms of the relation between the plastic increment of void ratio $\Delta e^{p}$ and the pressure parameter $p_{c}$,

$$
-\frac{\Delta e^{p}}{e_{0}}=a_{1} \exp \left(-\frac{\Lambda_{1}}{p_{c}}\right)+a_{2} \exp \left(-\frac{\Lambda_{2}}{p_{c}}\right)
$$

where $a_{1}, a_{2}, \Lambda_{1}$ and $\Lambda_{2}$ are material (positive) constants. In particular, coefficients $-e_{0} a_{1}$ and $-e_{0} a_{2}$ denote the increment of void ratio that would be achieved at infinite pressure by each of the two processes of densification, so that $0<a_{1}+a_{2} \leq 1$. Coefficients $\Lambda_{1}$ and $\Lambda_{2}$, having the dimension of stress, indicate the magnitude of the pressure at which the particular process of deformation has the maximum probability density.

Assuming incompressibility of the grain constituent:10, the plastic volumetric defor-

\footnotetext{
${ }^{10}$ Ceramic powders are usually obtained through spray-drying and are formed by granules with dimensions ranging between 50 and $200 \mu \mathrm{m}$, coated with the binder system. Granules are itself aggregates of crystals having dimensions on the order of $1 \mu \mathrm{m}$. The crystals are here assumed to be incompressible, while compressibility of granules is due to their internal voids.
} 
mation is related to the plastic void ratio increment according to the rule

$$
\Delta e^{p}=\left(1+e_{0}\right) \operatorname{tr} \boldsymbol{\epsilon}^{p},
$$

we get from eqn. (27)

$$
\operatorname{tr} \boldsymbol{\epsilon}^{p}=-\tilde{a}_{1} \exp \left(-\frac{\Lambda_{1}}{p_{c}}\right)-\tilde{a}_{2} \exp \left(-\frac{\Lambda_{2}}{p_{c}}\right),
$$

where $\tilde{a}_{i}=e_{0} a_{i} /\left(1+e_{0}\right), i=1,2$.

The hardening rule (29) is calibrated to describe uniaxial strain experiments, in which the permanent volumetric deformation has been measured at various forming pressure. In particular, taking the values $\Lambda_{1}=1.8 \mathrm{MPa}, \Lambda_{2}=40 \mathrm{MPa}, \tilde{a}_{1}=0.37, \tilde{a}_{2}=0.12$ gives the excellent interpolation presented in Fig. 12, where the volumetric plastic strain $\operatorname{tr} \epsilon^{p}$ is reported versus the hardening parameter $p_{c}$. It may be noted that we restrict the

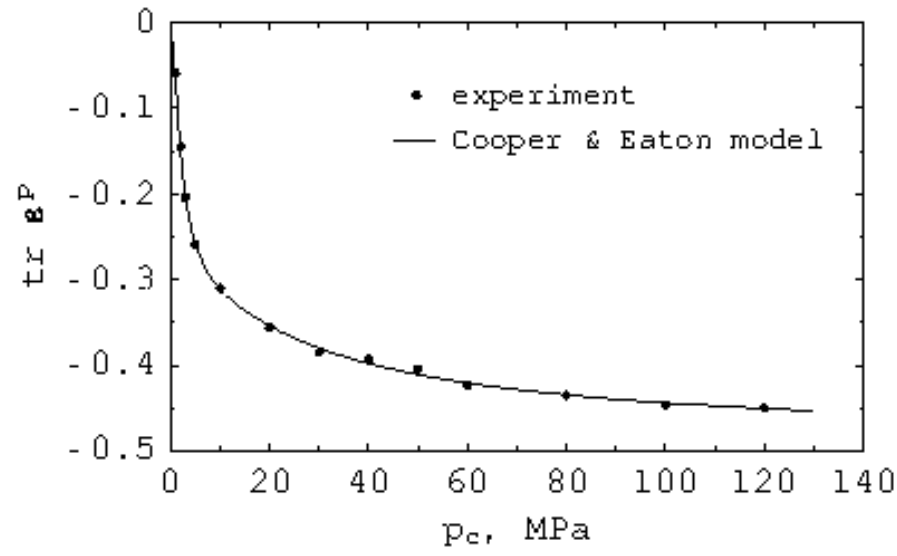

Figure 12: Plastic volumetric strain as a function of the forming pressure $p_{c}$. Experimental data relative to M KMS-96 alumina powder are compared with the model by Cooper and Eaton (1962) (solid line).

attention to the double-exponential law (29) for simplicity, but it would be certainly not difficult to include more complicated relationships, which - as suggested by Cooper and Eaton - could include an arbitrary number of exponentials and therefore describe also the third phase of compaction behaviour.

Eqn. (29) defines an implicit relation between the plastic deformation $\operatorname{tr} \boldsymbol{\epsilon}^{p}$ and the forming pressure $p_{c}$, which becomes explicit in terms of rates. In particular, the rate of eqn. (29) gives

$$
\dot{p}_{c}=-\frac{p_{c}^{2}}{\tilde{a}_{1} \Lambda_{1} \exp \left(-\frac{\Lambda_{1}}{p_{c}}\right)+\tilde{a}_{2} \Lambda_{2} \exp \left(-\frac{\Lambda_{2}}{p_{c}}\right)} \operatorname{tr} \dot{\boldsymbol{\epsilon}}^{p}
$$


providing the first hardening rule.

\subsubsection{Modelling the increase of cohesion}

Concerning the dependence of cohesion $c$ on the forming pressure $p_{c}$, we could recourse to models of adhesion between particles. We note that the celebrated JKR model (Johnson et al. 1971) (and also variants like for instance the DMT model) is not applicable in our case, since the ceramic granules considered here are highly plastic and the adhesion force cannot be treated as independent of the granule deformation. It seems more appropriate to recourse to the Bowden and Tabor (1950) model for adhesion between surfaces in contact. In this model, the cohesion depends on the real contact area and therefore on the normal pressure. Assuming a Herzian contact between spheres and that the cohesion $c$ is a linear function of the contact area, the following relationship

$$
c \sim p^{2 / 3},
$$

is found, in which $p$ is the contact pressure. The major concern with condition (31) is that it does not predict a limit for the increase of adhesion with pressure, which is a clear experimental evidence. More in detail, our experimental results reported in Fig. 3 can be manipulated employing our model and expressed in terms of cohesion $c$ versus the forming pressure $p_{c}$. The results are shown in Fig. 13, where interpolation using the Bowden and Tabor approximation (31) is also reported (dashed). The solid line in the figure is obtained employing the following law

$$
c=c_{\infty}\left[1-\exp \left(-\Gamma<p_{c}-p_{c b}>\right)\right],
$$

where $p_{c b}$ is the breakpoint pressure, $c_{\infty}$ and $\Gamma$ are two positive material parameters, the former defining the limit value of cohesion reached after substantial plastic deformation, the latter related to the 'velocity of growth' of cohesion. The values $\Gamma=0.026 \mathrm{MPa}^{-1}$, $c_{\infty}=2.3 \mathrm{MPa}$, and $p_{c b}=3.2 \mathrm{MPa}$ have been found to provide an excellent interpolation to experimental data. Note that the Macaulay bracket is needed since the cohesion is null before the threshold value defined by the breakpoint pressure is attained.

Taking now the rate of eqn. (32) and using (30) yields

$$
\dot{c}=\xi_{2} \operatorname{tr} \dot{\boldsymbol{\epsilon}}^{p},
$$

where

$$
\xi_{2}=-\frac{c_{\infty} \Gamma H\left(p_{c}-p_{c b}\right) \exp \left[-\Gamma\left(p_{c}-p_{c b}\right)\right] p_{c}^{2}}{\tilde{a}_{1} \Lambda_{1} \exp \left(-\frac{\Lambda_{1}}{p_{c}}\right)+\tilde{a}_{2} \Lambda_{2} \exp \left(-\frac{\Lambda_{2}}{p_{c}}\right)},
$$

providing the second hardening rule. Note that symbol $H$ in eqn. (34) denotes the Heaviside step function (defined for every scalar $\alpha$ as $H(\alpha)=1$, if $\alpha \geq 0, H(\alpha)=0$ otherwise). 


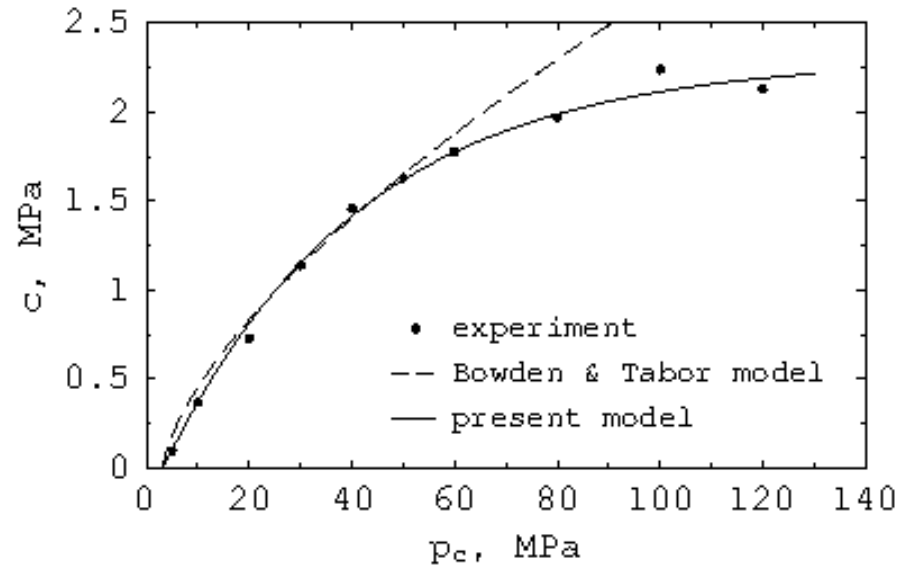

Figure 13: Variation of the cohesion $c$ as a function of the forming pressure $p_{c}$. Experimental results relative to M KMS-96 alumina powder are compared to our model (32) (solid line) and to that by Bowden and Tabor (1950) (dashed line).

\subsection{The elastoplastic coupling in rate form}

Taking the time derivative of eqns. (26) and (201) and considering eqn. (30) we get the dependence of elastic parameters on plastic strain in rate form

$$
\dot{d}=\xi_{3} \operatorname{tr} \dot{\boldsymbol{\epsilon}}^{p}, \quad \text { and } \quad \dot{\mu}=\xi_{4} \operatorname{tr} \dot{\boldsymbol{\epsilon}}^{p},
$$

where

$$
\begin{gathered}
\xi_{3}=-\frac{B H\left(p_{c}-p_{c b}\right) p_{c}^{2}}{\tilde{a}_{1} \Lambda_{1} \exp \left(-\frac{\Lambda_{1}}{p_{c}}\right)+\tilde{a}_{2} \Lambda_{2} \exp \left(-\frac{\Lambda_{2}}{p_{c}}\right)}, \\
\xi_{4}=\left(d-\frac{1}{d}\right) \mu_{1} \xi_{2}+c\left(1+\frac{1}{d^{2}}\right) \mu_{1} \xi_{3} .
\end{gathered}
$$

Employing the evolution equations (33) and (35) into the stress rate equations (22), we may write

$$
\dot{\boldsymbol{\sigma}}=\mathbb{E}\left[\dot{\boldsymbol{\epsilon}}^{e}\right]+\mathbb{P}\left[\dot{\boldsymbol{\epsilon}}^{p}\right],
$$

where the fourth-order tensor $\mathbb{P}$ defines the contribution of the elastoplastic coupling, in the sense that $\mathbb{P}$ is null in the usual, uncoupled plasticity and is defined as

$$
\mathbb{P}=\xi_{5} \boldsymbol{I} \otimes \boldsymbol{I}+2 \xi_{4} \boldsymbol{\epsilon}^{e} \otimes \boldsymbol{I},
$$


in which

$$
\begin{aligned}
\xi_{5}=-\frac{2}{3} \xi_{4} \operatorname{tr} \boldsymbol{\epsilon}^{e}+\xi_{2}\left[1+\left(d-\frac{1}{d}\right) \frac{\operatorname{tr} \boldsymbol{\epsilon}^{e}}{\tilde{\kappa}}-\exp \left(-\frac{\operatorname{tr} \boldsymbol{\epsilon}^{e}}{d^{1 / n} \tilde{\kappa}}\right)\right] \\
+\xi_{3} \frac{p_{0}+c}{\tilde{\kappa}} \operatorname{tr} \boldsymbol{\epsilon}^{e}\left[1+\frac{1}{d^{2}}-\frac{1}{n d^{1+1 / n}} \exp \left(-\frac{\operatorname{tr} \boldsymbol{\epsilon}^{e}}{d^{1 / n} \tilde{\kappa}}\right)\right]
\end{aligned}
$$

when elastoplastic coupling occurs.

Introducing now the 'irreversible' (in an infinitesimal stress cycle) strain rate

$$
\dot{\boldsymbol{\epsilon}}^{i}=\mathbb{G}\left[\dot{\boldsymbol{\epsilon}}^{p}\right]
$$

in which

$$
\mathbb{G}=\boldsymbol{I} \underline{\bar{\otimes}} \boldsymbol{I}-\mathbb{E}^{-1} \mathbb{P}=\boldsymbol{I} \underline{\bar{\otimes}} \boldsymbol{I}+\xi_{6} \boldsymbol{I} \otimes \boldsymbol{I}+\xi_{7} \boldsymbol{\epsilon}^{e} \otimes \boldsymbol{I},
$$

and

$$
\xi_{6}=-\frac{\xi_{5}}{3 K_{t}}-\frac{2 \mu-3 K_{t}}{9 \mu K_{t}} \xi_{4} \operatorname{tr} \boldsymbol{\epsilon}^{e}, \quad \xi_{7}=-\frac{\xi_{4}}{\mu},
$$

we may transform the rate equation (37) into the equivalent form

$$
\dot{\boldsymbol{\sigma}}=\mathbb{E}[\dot{\boldsymbol{\epsilon}}]-\mathbb{E}\left[\dot{\boldsymbol{\epsilon}}^{i}\right] .
$$

Since tensor $\mathbb{G}$ is assumed positive definite, implying that

$$
\dot{\boldsymbol{\epsilon}}^{i} \cdot \dot{\boldsymbol{\epsilon}}^{p}>0
$$

the inverse of $\mathbb{G}$ is given by

$$
\mathbb{G}^{-1}=\boldsymbol{I} \underline{\bar{\otimes}} \boldsymbol{I}+\xi_{8} \boldsymbol{I} \otimes \boldsymbol{I}+\xi_{9} \boldsymbol{\epsilon}^{e} \otimes \boldsymbol{I},
$$

where

$$
\xi_{8}=-\frac{\xi_{6}}{1+3 \xi_{6}+\xi_{7} \operatorname{tr} \boldsymbol{\epsilon}^{e}}, \quad \xi_{9}=-\frac{\xi_{7}}{1+3 \xi_{6}+\xi_{7} \operatorname{tr} \boldsymbol{\epsilon}^{e}} .
$$

The irreversible deformation rate $\dot{\boldsymbol{\epsilon}}^{i}$ defined by eqn. (40) is the rate of deformation which is not recovered in an infinitesimal stress cycle. This should not be confused with the plastic deformation rate $\dot{\boldsymbol{\epsilon}}^{p}$, which can be only detected upon unloading at zero stress. This concept is illustrated in Fig. 14 with reference to a hypothetical volumetric stress/strain law, where the rate deformation at unloading $\mathbb{E}^{-1}[\dot{\boldsymbol{\sigma}}]$ is also indicated. Due to the plastic increase in elastic stiffness, we note that (22) implies that the plastic $\dot{\boldsymbol{\epsilon}}^{p}$ and inelastic $\dot{\boldsymbol{\epsilon}}^{i}$ rates are different. 


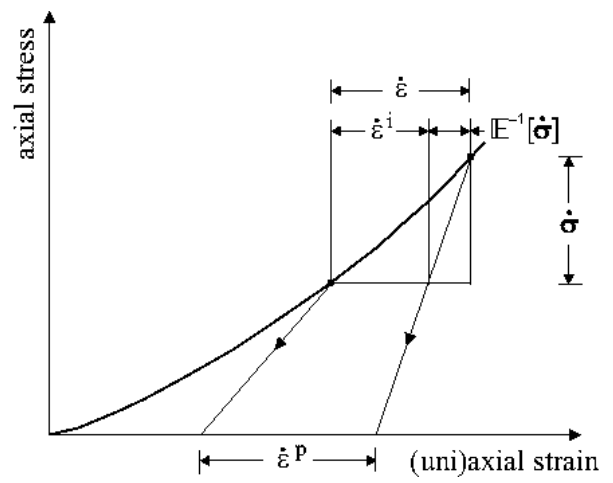

Figure 14: Irreversible, and plastic rate deformations, with reference to volumetric deformation.

\subsection{Flow rule}

A crucial point is now the definition of the flow rule, that following Bigoni (2000) is assumed to govern the irreversible strain rate (instead of the plastic, see Gajo et al. 2004 for a discussion on this issue)

$$
\dot{\boldsymbol{\epsilon}}^{i}=\dot{\lambda} \boldsymbol{P}
$$

Experimental evidence for granular material supports the use of a deviatoric associative flow rule, which is adopted here. Therefore, tensor $\boldsymbol{P}$ is related to the yield function gradient $\boldsymbol{Q}$ through

$$
\boldsymbol{P}=\boldsymbol{Q}-\frac{\epsilon(1-\Phi) \operatorname{tr} \boldsymbol{Q}}{3} \boldsymbol{I}, \quad 0 \leq \epsilon \leq 1,
$$

where $\epsilon$ is a parameter governing the entity of volumetric nonassociativity, so that $\epsilon=0$ gives the associative flow rule. All indirect evidences point to flow rule nonassociativity for ceramic powders, so that we feel that eqn. (48) is appropriate; however, experimental evidence for alumina powder is not available and associativity will be assumed for simplicity in the following numerical simulation.

The rate constitutive equations can now be obtained via Prager's consistency, so that $\dot{F}=0$ during plastic deformation. Imposing this condition suggests the following definition of hardening modulus

$$
h=-\frac{1}{\dot{\lambda}}\left(\frac{\partial F}{\partial p_{c}} \dot{p}_{c}+\frac{\partial F}{\partial c} \dot{c}\right)
$$

which is positive in the case of hardening, negative for softening and null for ideally plastic behaviour. The derivatives of $F$ with respect to the hardening parameters $p_{c}$ and 
$c$ appearing in eqn. (49) are given by

$$
\begin{aligned}
\frac{\partial F}{\partial p_{c}} & =-M \sqrt{\left(\Phi-\Phi^{m}\right)[2(1-\alpha) \Phi+\alpha]} \\
& +M \frac{p_{c}(p+c)}{\left(p_{c}+c\right)^{2}} \frac{\left(1-m \Phi^{m-1}\right)[2(1-\alpha) \Phi+\alpha]+2(1-\alpha)\left(\Phi-\Phi^{m}\right)}{2 \sqrt{\left(\Phi-\Phi^{m}\right)[2(1-\alpha) \Phi+\alpha]}}
\end{aligned}
$$

and

$$
\begin{aligned}
\frac{\partial F}{\partial c}=-M & \frac{p_{c}\left(p_{c}-p\right)}{\left(p_{c}+c\right)^{2}} \\
& \cdot \frac{\left(1-m \Phi^{m-1}\right)[2(1-\alpha) \Phi+\alpha]+2(1-\alpha)\left(\Phi-\Phi^{m}\right)}{2 \sqrt{\left(\Phi-\Phi^{m}\right)[2(1-\alpha) \Phi+\alpha]}}
\end{aligned}
$$

\subsection{The final rate equations}

Employing definition (49) into Prager's consistency yields the elastoplastic rate equations

$$
\dot{\boldsymbol{\sigma}}= \begin{cases}\mathbb{E}[\dot{\boldsymbol{\epsilon}}]-\frac{<\mathbf{Q} \cdot \mathbb{E}[\dot{\boldsymbol{\epsilon}}]>}{h+\boldsymbol{Q} \cdot \mathbb{E}[\boldsymbol{P}]} \mathbb{E}[\mathbf{P}] & \text { if } F\left(\boldsymbol{\sigma}, p_{c}, c\right)=0, \\ \mathbb{E}[\dot{\boldsymbol{\epsilon}}] & \text { if } F\left(\boldsymbol{\sigma}, p_{c}, c\right)<0 .\end{cases}
$$

It may be noted that the elastoplastic tangent operator becomes symmetric in the specific case of the associative flow rule, $\boldsymbol{P}=\boldsymbol{Q}$.

\section{$3 \quad$ Numerical simulations}

The proposed constitutive model was implemented into UMAT, the subroutine available within the commercial finite element code ABAQUS (Ver. 6.3; Hibbitt, Karlsson \& Sorensen, 2002, Pawtucket, RI, USA). The employed numerical integration scheme was the so-called 'cutting-plane algorithm', proposed by Simo and Ortiz (1985), Ortiz and Simo (1986), Simo and Huges (1987). A full Newton-Rapson scheme has been employed for the solution of the nonlinear finite element problem. Parameters of the models employed for the simulations are summarized in Table 1.

\subsection{Forming of tablets}

Simulations of uniaxial deformation of cylindrical samples, or, in other words, forming of tablets, are reported in Fig. 15, together with our experimental results, marked by spots. In the figure the applied vertical load (in $\mathrm{kN}$ ) is reported versus the vertical displacement (in $\mathrm{mm}$ ) and different forming pressures, equal to $\{60,80,100,120\} \mathrm{MPa}$, have been considered. The fact that the experiments are correctly simulated employing 
Table 1: Values of material parameters estimated from experiments for alumina powder 392 Martoxid KMS-96.

\begin{tabular}{||c||}
\hline \hline Material parameters defining the yield surface \\
$m=2, \alpha=0.1, \beta=0.19, \gamma=0.9, M=1.1$ \\
\hline Elastic logarithmic bulk modulus $\kappa=0.04$ \\
\hline Material parameters defining the hardening rule (29) \\
$\Lambda_{1}=1.8 \mathrm{MPa}, \Lambda_{2}=40 \mathrm{MPa}, \tilde{a}_{1}=0.37, \tilde{a}_{2}=0.12$ \\
\hline Material parameters defining the hardening rule (32) \\
$\Gamma=0.026 \mathrm{MPa}^{-1}, c_{\infty}=2.3 \mathrm{MPa}, p_{c b}=3.2 \mathrm{MPa}$ \\
\hline Material parameters defining coupling rules (26) and (20) \\
$B=0.18 \mathrm{MPa}^{-1}, n=6, \mu_{0}=1 \mathrm{MPa}, \mu_{1}=64$ \\
\hline Material parameter defining the flow rule $\epsilon=0$ \\
\hline \hline
\end{tabular}
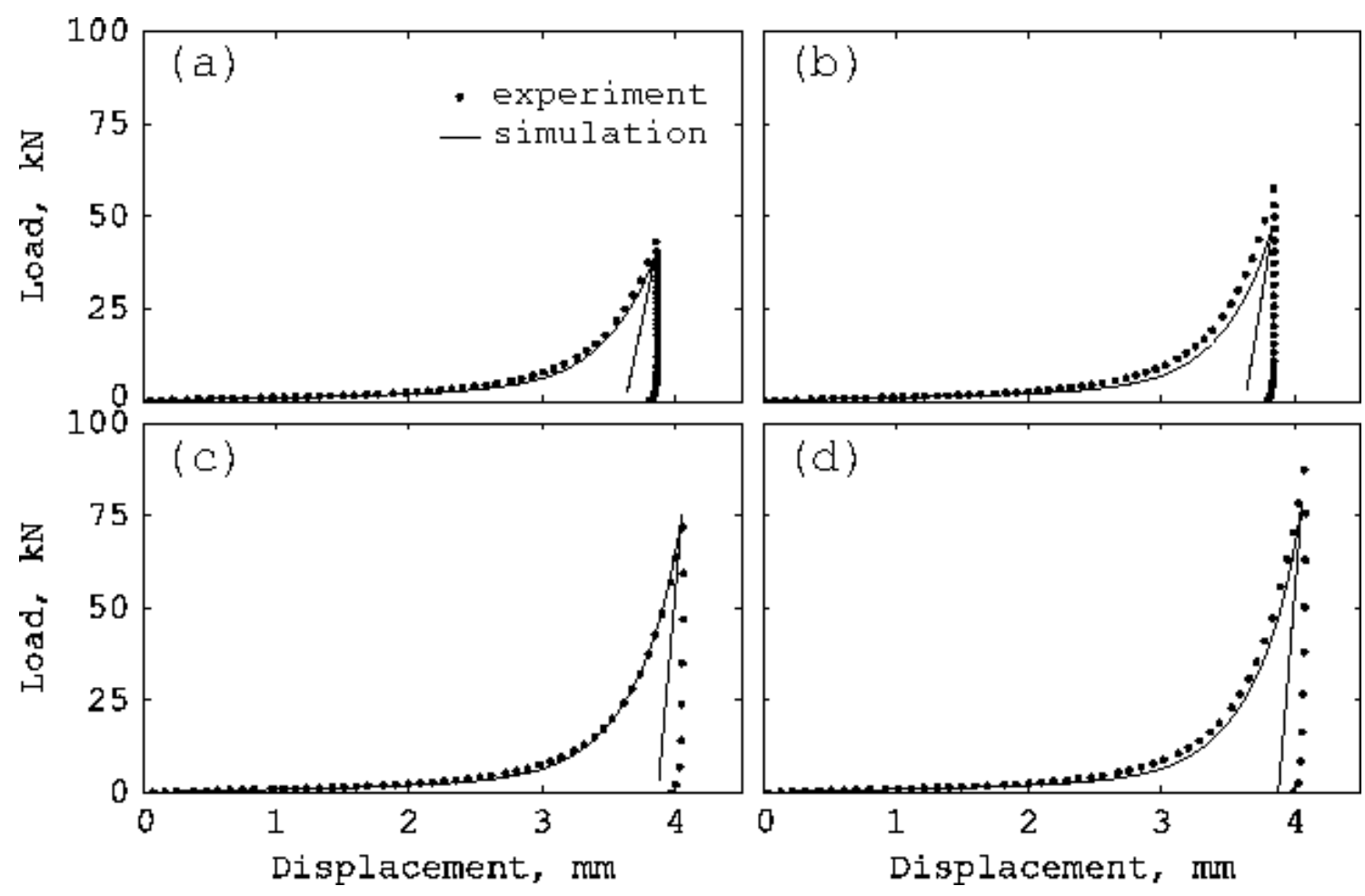

Figure 15: Experimental (data relative to M KMS-96 alumina powder) and simulated load vs. displacement curves for tablets formed at various final pressures: 60 (a), 80 (b), 100 (c), and $120 \mathrm{MPa}$ (d). Different values of $n$ have been considered.

the finite element discretisation should be considered as a succesful feedback on the 'consistency' of the model and its implementation. Moreover, we note that the model describes the progressive increase in elastic stiffness (visible at unloading) in qualitative 
and quantitative agreement with experimental data.

\subsection{Forming of a simple ceramic piece}

Numerical simulations were performed to describe forming of the (axisymmetric) piece geometrically described in Fig. 16 and shown as a green body after forming in Figs. 16,

In particular, four pieces have been formed at a final mean pressure of $100 \mathrm{MPa}$,

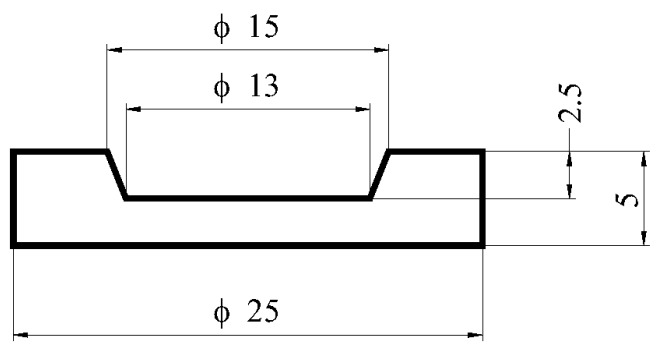

Figure 16: Geometry of the formed piece (dimensions in $\mathrm{mm}$ ).

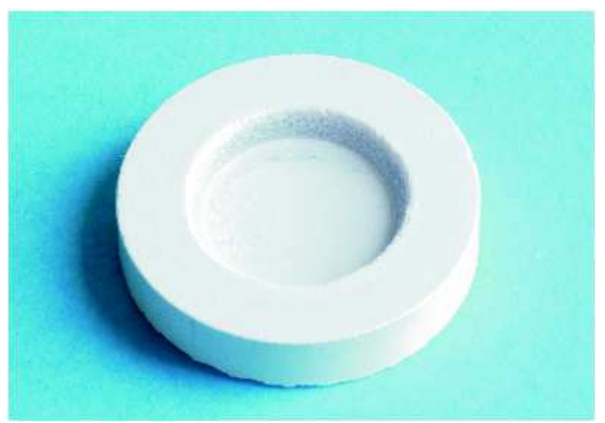

Figure 17: Photograph of the formed green piece ( $5 \mathrm{~g}$ of M KMS-96 alumina powder has been used and a final mean pressure of $100 \mathrm{MPa}$ has been reached).

starting from $5 \mathrm{~g}$ of the M KMS-96 alumina powder employed in all our experiments. The axisymmetric mesh, employing 4-node elements (CAX4), used in the simulations is shown in Fig. 18,

The following assumptions have been introduced to simulate the entire forming process:

- the die is rigid; 


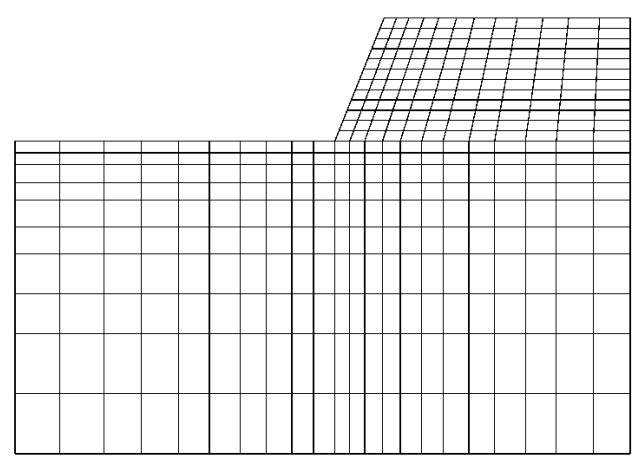

Figure 18: Initial mesh.

- friction is neglected at the contact between powder and die walls;

- the initial configuration is that shown meshed in Fig. 18.

After the initial state, defined by given initial values of void ratio and confining pressure $\left(e_{0}=2.129\right.$ and $p_{0}=0.063 \mathrm{MPa}$ have been assumed, respectively), has been prescribed, the loading history is assigned, in terms of the following three sequential steps:

1. forming is prescribed by imposing the motion of the upper part of the boundary ( $3.78 \mathrm{~mm}$, corresponding to the value measured during forming at the final load of $50 \mathrm{kN})$;

2. unloading is simulated by prescribing null forces on the upper part of the boundary;

3. ejection is simulated by prescribing null forces on all the boundary.

The final deformed mesh (at the end of step 3), is reported in Fig. 19 superimposed on the initial mesh. It can be noted from the figure that the elements near the corner of the punch are excessively distorted so that results in this zone should be considered unrealistic. Comparing the meshes, it can be observed that the deformation suffered by the piece is moderately large.

The hydrostatic stress component $p$ (taken positive when compressive, upper part in the figure), the Mises stress $q$ (central part in the figure) and the void ratio $e$ (lower part in the figure) are reported in Fig. 20 at the end of step 1, in Fig. 21 at the end of step 2, and in Fig. 22 at the end of step 3.

Regarding Fig. 20 (representative of step 1) we may note that, excluding the small, unrepresentative zone near the corner of the punch, the hydrostatic stress $p$ ranges between 40 and $100 \mathrm{MPa}$ and the Mises stress $q$ between 25 and $120 \mathrm{MPa}$, evidencing a high stress inhomogeneity. 


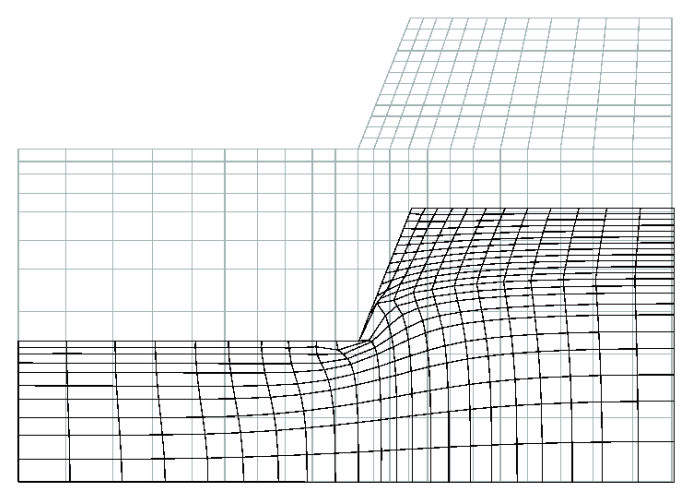

Figure 19: Initial and deformed (step 3) meshes.

Considering Fig. 21 (representative of step 2) it may be important to note that residual stress is definitely high, due to the lateral constraint (provided by the walls of the mold) still present at the end of step 2. The knowledge of the lateral stress is important for practical purposes since the force needed for the ejection of the final piece can be estimated from this value employing the Coulomb friction law. In particular, a rough, but simple evaluation can be immediately obtained from numerical output at the end of step 2 employing the formula

ejection force $=\alpha \tan \phi($ mean lateral stress $\times$ lateral surface of the piece $)$,

where $\phi$ is the powder friction angle (equal to $32^{\circ}$ in our case) and $\alpha$ is a coefficient dependent on the roughness of the die wall and ranging between 0 and 1 .

Regarding Fig. 22 (representative of step 3), we note that $p$ and $q$ represent, in terms of hydrostatic stress and Mises stress components, the residual stress distribution in the green body at the end of forming.

Excluding again the unrepresentative zone near the corner of the punch, the hydrostatic stress $p$ ranges now between -1.5 and $6 \mathrm{MPa}$ and the Mises stress $q$ between 1 and $6 \mathrm{MPa}$. Moreover, the void ratio varies between 0.6 and 0.9 . It can be noted that the minimum void ratio is not associated with the maximum residual mean stress, it is rather associated with the maximum mean stress reached during loading (step 1). The results suggest also that two oblique zones of material are formed, the outer of which is subject to high compressive mean stresses, whereas the inner is subject to tensile stresses, creating a sort of truss-like mechanism. This can be considered representative of a situation where the tensile stresses tend to open possible microcracks, leading to defects formation in the green. It is however worth remembering that, even in the absence of macro defects, the mechanical behaviour of the green and the shrinkage during future sintering are deeply affected by the inhomogeneities in the residual stress and density distributions. 

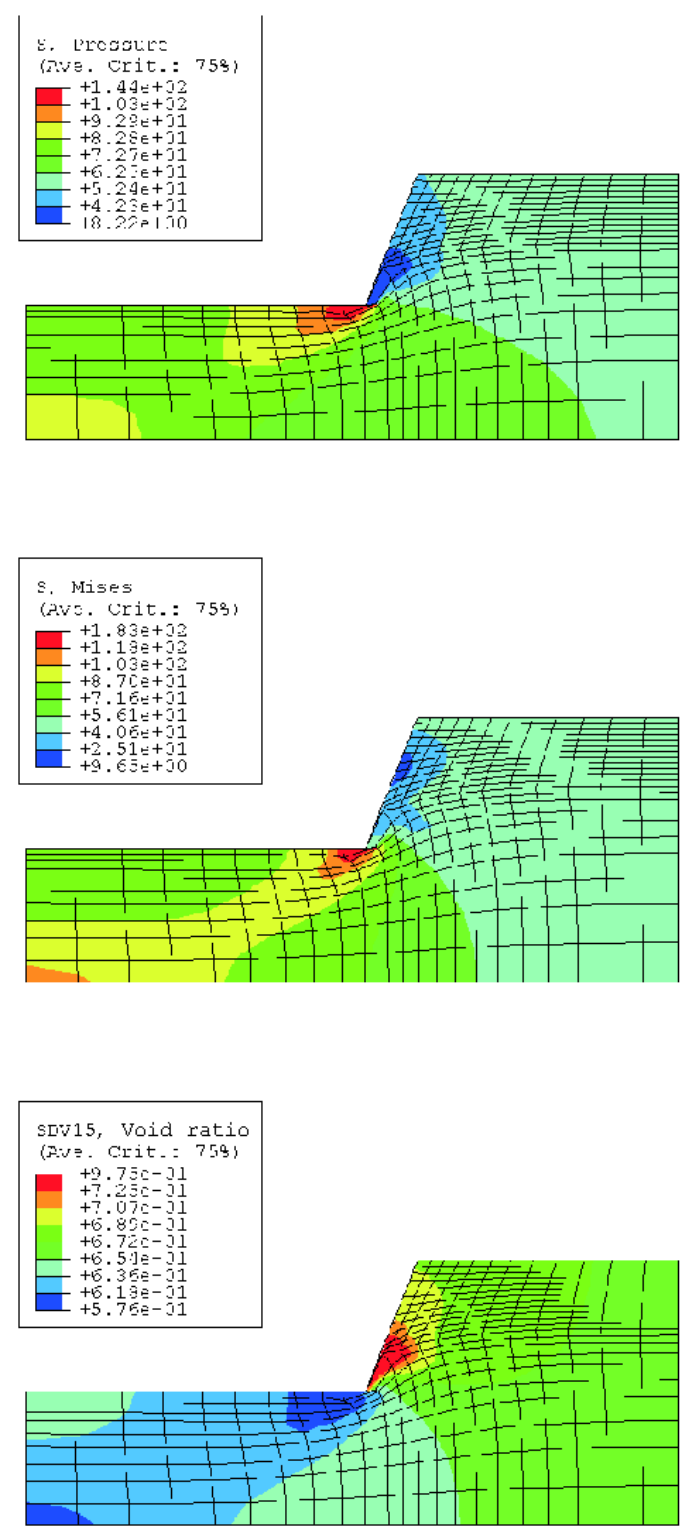

Figure 20: Hydrostatic stress $p$ (MPa, upper part), Mises stress $q$ (MPa, central part), and Void ratio $e$ (lower part) distributions at the end of step 1 (after loading). 

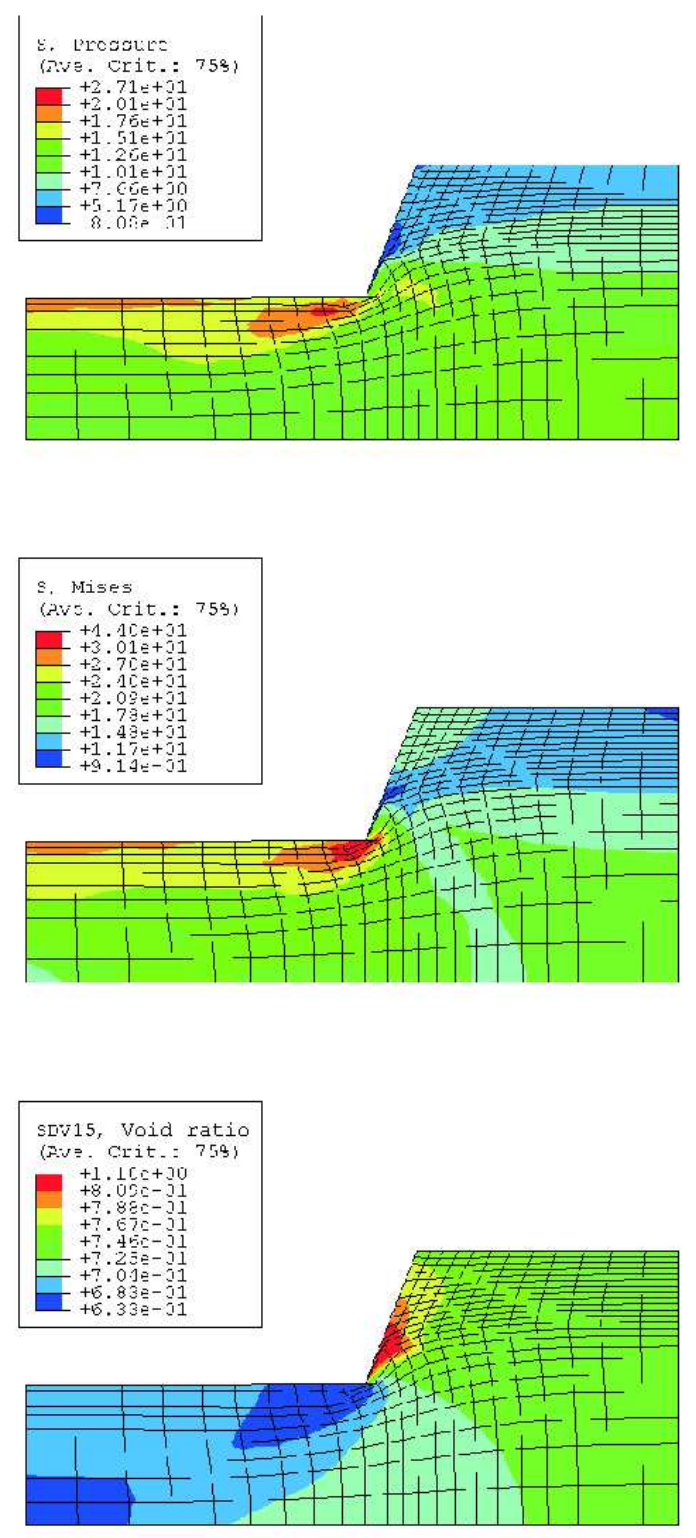

Figure 21: Hydrostatic stress $p$ (MPa, upper part), Mises stress $q$ (MPa, central part), and Void ratio $e$ (lower part) distributions at the end of step 2 (after punch removal). 

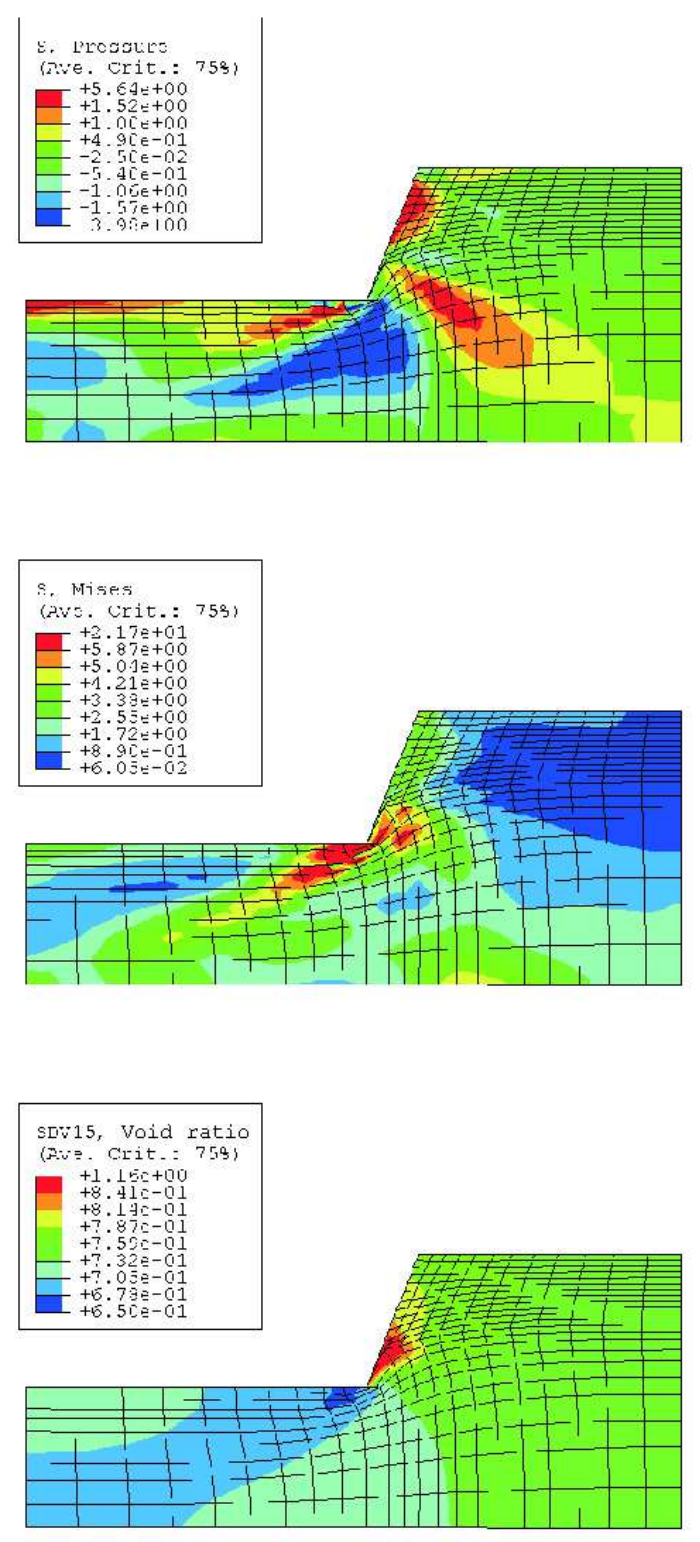

Figure 22: Hydrostatic stress $p$ (MPa, upper part), Mises stress $q$ (MPa, central part), and Void ratio $e$ (lower part) distributions at the end of step 3 (in the green piece). 
From the comparison reported in Fig. 19 between the initial mesh and that at the end of step 3, it becomes now possible to evaluate the springback effect. In contrast to the prediction of the simple model employed by Piccolroaz et al. (2002), it can be noted that now the model correctly predicts that the springback effect and the shape distortion are very small. In particular, the final diameter of the piece is estimated to be $0.1 \mathrm{~mm}$ larger than the inner diameter of the die, in agreement with our experimental observations.

The cohesion $c$ attained by the material at the end of the overall process is shown in Fig. 23, upper part, whereas the elastic properties of the final piece are reported in Fig. 23 (central and lower parts, respectively), in terms of tangent bulk modulus $K_{t}$ and shear modulus $\mu$. The inhomogeneity of the elastic properties evidenced in Fig. 23 is obviously a consequence of elastoplastic coupling. This effect and also the increase in cohesion have been not modelled in the simple analysis presented by Piccolroaz et al. (2002). It may be observed by comparing the maps shown in Fig. 23 with the map of hydrostatic stress $p$ at the end of step 1 (Fig. 20, upper part) that there is a strong relation between mechanical properties gained by the material in the final piece and the mean stress reached during loading. This results from our analyses to represent the most important parameter in the entire forming process.

Experimental and simulated load displacement curves during forming (natural and semilogarithmic representations are reported) are compared in Fig. 24, together with the results obtained by Piccolroaz et al. (2002) included (dashed) in the figure. Beyond the excellent agreement, it may be instructive to compare the present numerical simulation with that performed by Piccolroaz et al. (2002) employing a simple model. First, we note that the simple model approximately describes two straight lines, whereas the present model describes a curved line, much closer to the experimental results. Second, we may speculate on the limits of the approach presented by Piccolroaz et al. (2002) and of many similar models available in the literature; in particular, the modelling can be accurate enough, if intended to predict a 'global' force-displacement curve like that reported in Fig. 24. On the other hand, the simple model does not predict increase in cohesion and dependence of elastic properties on plastic deformation, so that the internal stress and strain distributions result almost completely different.

A photograph of the lower side of the formed pieces is shown in Fig. 25, where we can note the formation of annular dark zones, evidencing a sliding between material and mold. This sliding is indeed predicted by the simulations, so that the radial displacement at the mold contact is reported in Fig. 26, superimposed to the photograph of one of the pieces shown in Fig. 25, so that, since the dark zone corresponds to the peak of the radial displacement, simulations again agree with experimental observations.

\subsection{The effects of large strains}

In order to have an insight on the possible effects of large strains, a few analyses have been performed using the option NLGEOM available on ABAQUS, still employing the 

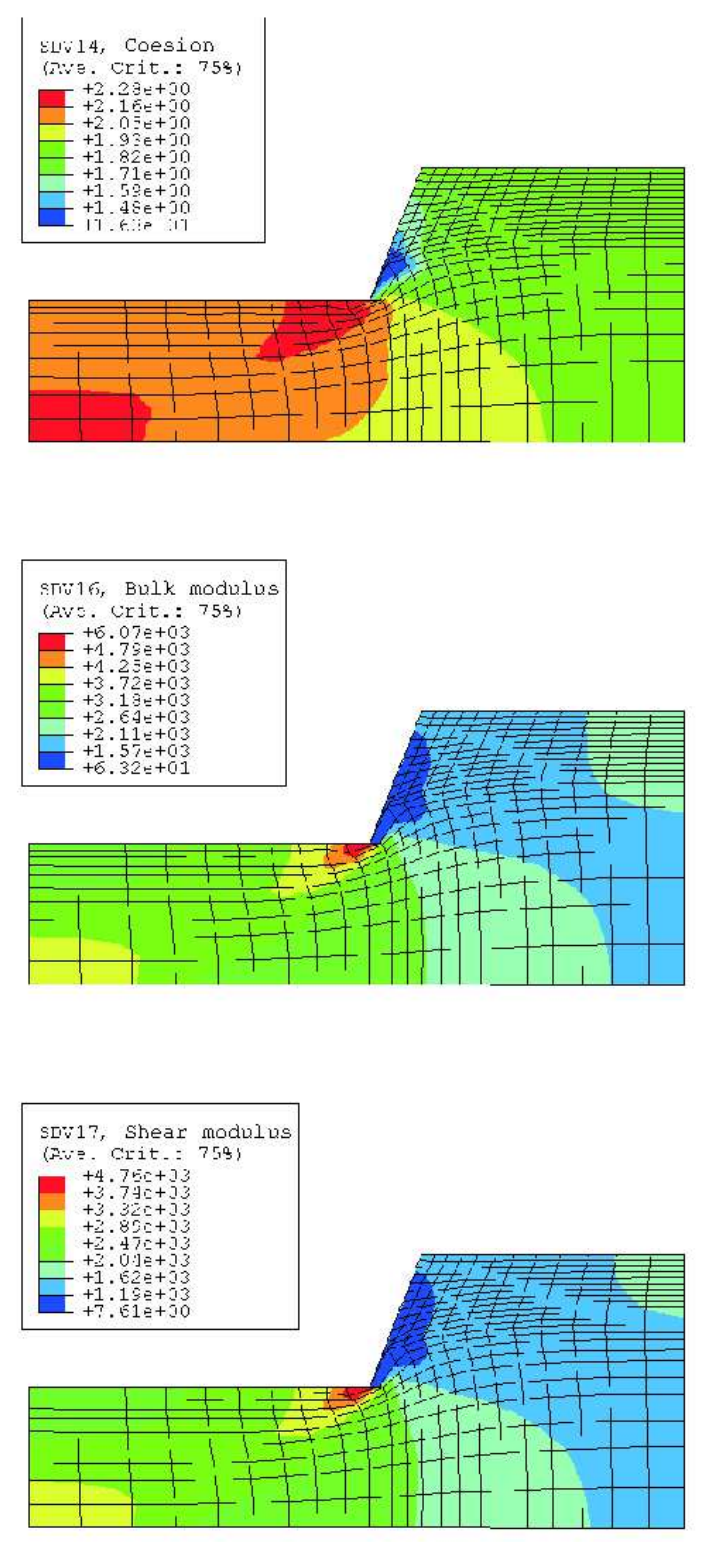

Figure 23: Distribution of cohesion $c$ (MPa, upper part), bulk modulus $K_{t}$ (MPa, central part), and shear modulus $\mu$ (MPa, lower part) at the end of step 3 (in the green piece). 

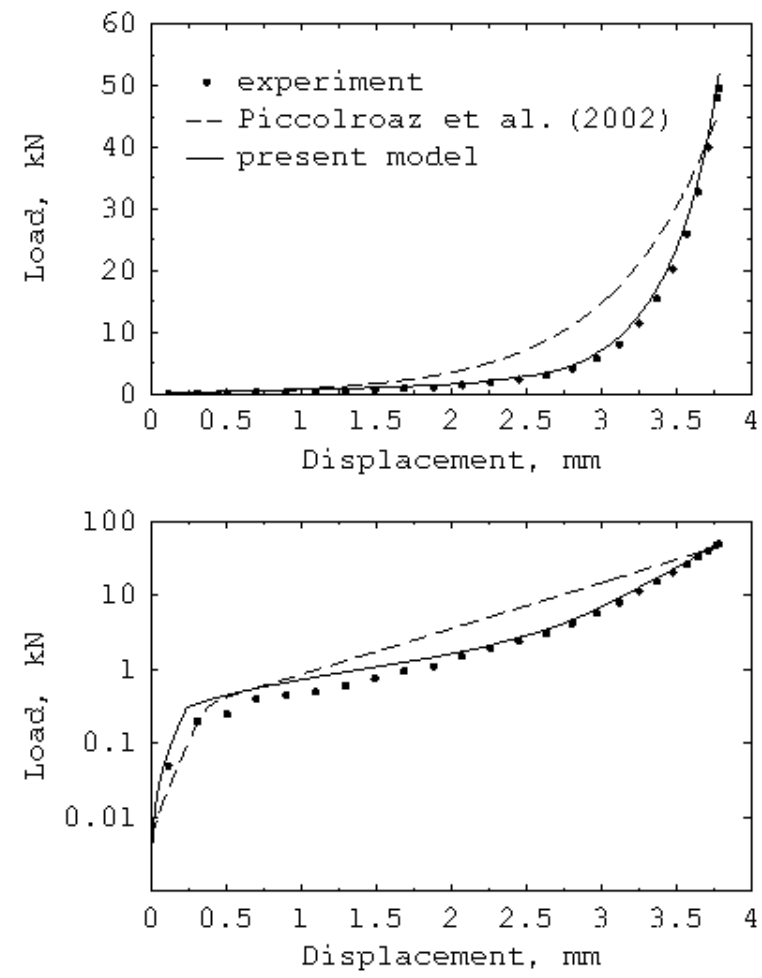

Figure 24: Experimental (M KMS-96 alumina powder) and simulated load vs. displacement curves during forming of the piece shown in Fig. 17, in natural and semilog representations. Results by Piccolroaz et al. (2002) are also reported (dashed).

presented small-strain formulation. A representative result is shown dashed in Fig. 27, relative to the forming of a tablet [see Fig. 15 (d)]. Obviously, a rigorous analysis would require a complete model reformulation and reinterpretation of experimental results. However, we believe that due to the fact that the deformations are only moderately large and do not involve rotations, the simulation shown in Fig. 27 should at least give some understanding of the main differencies between predictions expected from the two formulations. We note that the effect of geometrical nonlinearities yields, as expected, a stiffening of the response, but does not change the results qualitatively. However, the quantitative difference may be enough to suggest the interest in a large strain formulation, which is given in the Part II of this paper (Piccolroaz et al. 2005).

\section{Conclusions}

Results presented of this paper provide a constitutive framework to realistically describe forming processes of ceramic materials. Even if the experimental results are still in- 


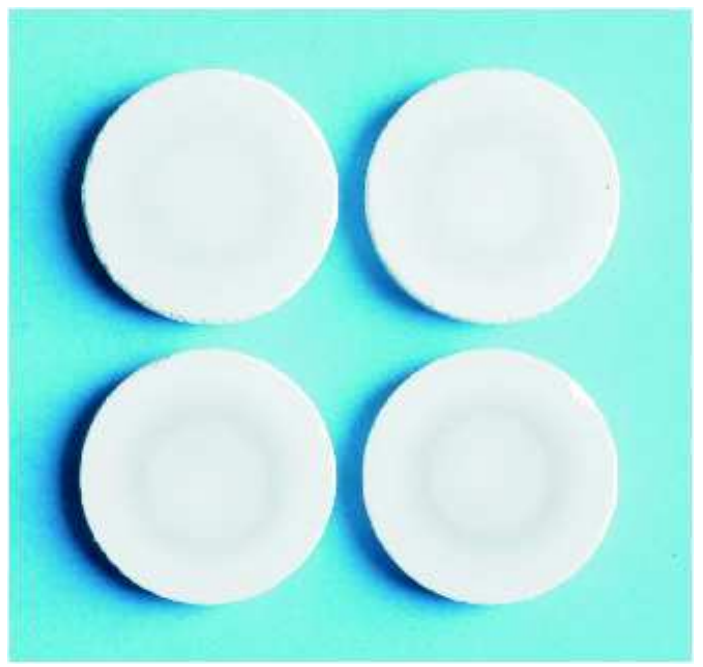

Figure 25: Photograph of the lower side of the formed pieces.

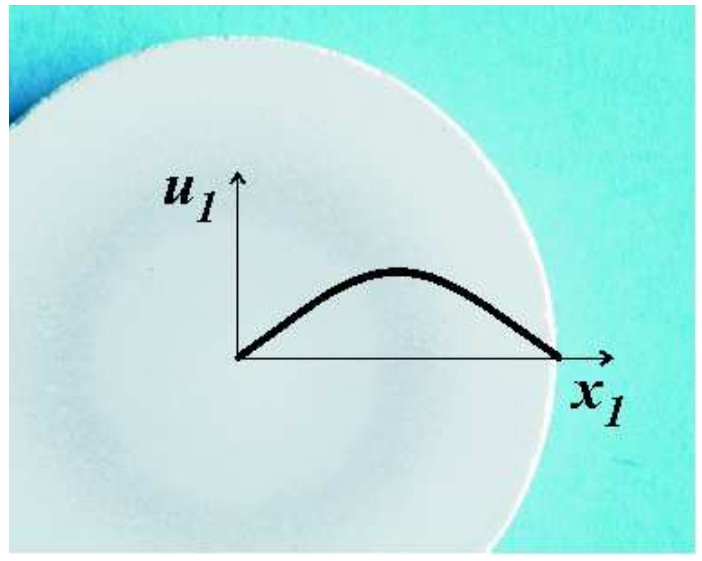

Figure 26: Simulated radial displacements superimposed to the photograph of the lower surface of the formed piece.

complete and the employed elastoplastic model has been developed in a small strain formulation, it has been shown that it is possible to predict:

- the force needed for mold ejection,

- the springback effect and related shape distortion of formed pieces,

- the residual stress distribution, 


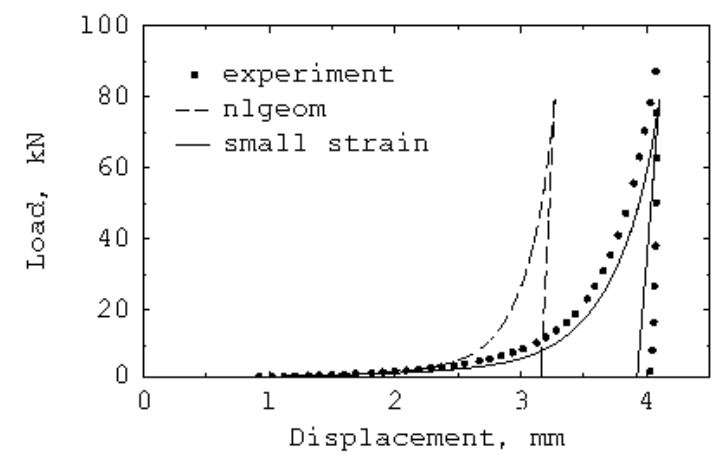

Figure 27: Experimental (M KMS-96 alumina powder) and simulated load vs. displacement curves for uniaxial strain. The prediction obtained employing the option NLGEOM has been reported (dashed) together with the prediction relative to the small strain assumption.

- the gain in cohesion and the final elastic properties,

- the density distribution and the related presence of defects in the green body.

The last of the above points is related to the prediction of defects in the sintered piece and therefore its investigation has an important consequences in the design of ceramic elements.

In closure, we mention that the present modelling can be extended in different directions. Introducing thermoplastic effects, the sintering phase might be covered by modelling, so that simulation could be extended to the entire production process. Moreover, both sintering aids and powder characteristics might enter the elastic-plastic constitutive laws, so that the optimal powder composition and morphology could be predicted for different forming problems.

\section{Acknowledgments}

Financial support of MIUR-COFIN 2003 'Fenomeni di degrado meccanico di interfacce in sistemi strutturali: applicazioni in Ingegneria Civile ed a campi di ricerca emergenti' is gratefully acknowledged. 


\section{References}

[1] Ahzi, S., Asaro, R.J. and Parks, D.M. (1993) Application of crystal plasticity theory for mechanically processed BSCCO superconductors. Mech. Materials 15, 201-222.

[2] Ariffin, A.K., Gethin, D.T. and Lewis, R.W. (1998) Finite element simulation and experimental validation for multilevel powder compact. Powder Metallurgy 41, 189-197.

[3] Aydin, I., Briscoe, B.J. and Sanliturk, K.Y. (1997a) Dimensional variation of diepressed ceramic green compacts: comparison of a finite element modelling with experiment. J. Eur. Ceram. Soc. 17, 1201-1212.

[4] Aydin, I., Briscoe, B.J. and Ozkan, N. (1997b) Modeling of powder compaction: a review. MRS Bull. 22, 45-51.

[5] Bigoni, D. (2000) Bifurcation and instability of non-associative elasticplastic solids. In CISM Lecture Notes No. 414, Material instabilities in elastic and plastic solids, Petryk, H. Ed., pp.1-52, Springer-Verlag, WienNew York.

[6] Bigoni, D. and Hueckel, T. (1991) Uniqueness and localization - I. Associative and non-associative elastoplasticity. Int. J. Solids Struct. 28, 197-213.

[7] Bigoni, D., Piccolroaz, A. (2004) Yield criteria for quasibrittle and frictional materials. Int. J. Solids Struct. 41, 2855-2878.

[8] Bigoni, D., Hueckel, T. (1991) Uniqueness and localization-II. Coupled elastoplasticity. Int. J. Solids Struct. 28, 215-224.

[9] Bowden, F.P. and Tabor, D. (1950) The friction and lubrication of solids. Oxford Science Publ., Clarendon Press.

[10] Brandt, J. and Nilsson, L. (1998) FE-simulation of compaction and solid-state sintering of cemented carbides. Mech. Cohesive-Frict. Mater. 3, 181-205.

[11] Brandt, J. and Nilsson, L. (1999) A constitutive model for compaction of granular media, with account for deformation induced anisotropy. Mech. Cohesive-Frict. Mater. 4, 391-418.

[12] Brown, S.B. and Abou-Chedid, G. (1994), Yield behaviour of metal powder assemblages, J. Mech. Phys. Solids 42, 383-399.

[13] Brown, S.B. and Weber, G.A. (1988) A constitutive model for the compaction of metal powders. Mod. Dev. Powder Metall. 18-21, 465-476.

[14] Capurso, M. (1979) Extremum theorems for the solution of the rate problem in elastic-plastic fracturing structures. J. Struct. Mech. 7, 411-434.

[15] Cooper, A.R. and Eaton, L.E. (1962) Compaction behavior of several ceramic powders. J. Am. Ceram. Soc. 45, 97-101.

[16] Deis, T.A. and Lannutti, J.J. (1998) X-ray computed tomography for evaluation of density gradient formation during the compaction of spray-dried granules. J. Am. Ceram. Soc. 81, 1237-1247. 
[17] Dougill, J.W. (1976) On stable progressively fracturing solids. Z. Angew. Math. Phys. 27, 423-437.

[18] Ewsuk, K.G. (1997) Compaction science and technology. MRS Bull. 22, 14-16.

[19] Ewsuk, K.G., Argüello, J.G., Zeuch, D.H., Farber, B., Carinci, L., Kaniuk, J., Keller, J., Cloutier, C., Gold, B., Cass, R.B., French, J.D., Dinger, B. and Blumenthal, W. (2001) CRADA develops model for powder pressing and die design, Part one. Am. Ceram. Soc. Bull. 80, 53-60.

[20] Ewsuk, K.G., Argüello, J.G., Zeuch, D.H., Farber, B., Carinci, L., Kaniuk, J., Keller, J., Cloutier, C., Gold, B., Cass, R.B., French, J.D., Dinger, B. and Blumenthal, W. (2001) CRADA develops model for powder pressing and die design, Part two. Am. Ceram. Soc. Bull. 80, 41-46.

[21] Gajo, A., Bigoni, D. and Wood, D.M. (2004) Multiple shear band development and related instabilities in granular materials. J. Mech. Phys. Solids 52, 2683-2724.

[22] German, R.M. (1984) Powder Metallurgy Science. MPIF, Princeton, New Jersey.

[23] Glass, S.J. and Ewsuk, K.G. (1997) Ceramic powder compaction. MRS Bull. 22, 24-28.

[24] Hausner, H.H. and Kumar-Mal, M. (1982) Handbook of Powder Metallurgy. Chemical Publishing.

[25] Hueckel, T., (1975) On plastic flow of granular and rock-like materials with variable elasticity moduli. Bull. Pol. Acad. Sci., Ser. Techn. 23, 405-414.

[26] Hueckel, T. (1976) Coupling of elastic and plastic deformation of bulk solids. Meccanica $11,227-235$.

[27] Hueckel, T. and Maier, G. (1977) Incremental boundary value problems in the presence of coupling of elastic and plastic deformations: a rock mechanics oriented theory. Int. J. Solids Struct. 13, 1-15.

[28] Jaky, J. (1944) The coefficient of earth pressure at Rest. J. Soc. Hungarian Arch. , 355-358.

[29] Johnson, K.L., Kendall, K. and Roberts, A.D. (1971) Surface energy at the contact of elastic solids. Proc. R. Soc. Lond. A 324, 301-313.

[30] Keller, J.M., French, J.D., Dinger, B., McDonough, M., Gold, B., Cloutier, C., Carinci, L., Van Horn, E., Ewsuk, K. and Blumenthal, B. (1998) Industry, government team to improve ceramic manufacturing. Am. Ceram. Soc. Bull. 77, 52-57.

[31] Khoei, A.R. and Lewis, R.W. (1999) Adaptive finite element remeshing in a large deformation analysis of metal powder forming. Int. J. Numer. Meth. Engng. 45, 801-820.

[32] Kim, H.G., Gillia, O., Dorémus, P. and Bouvard, D. (2002) Near net shape processing of a sintered alumina component: adjustment of pressing parameters through finite element simulation. Int. J. Mech. Sci. 44, 2523-2539. 
[33] Kim, K.T., Choi, S.W. and Park, H. (2000) Densification behavior of ceramic powder under cold compaction. ASME J. Eng. Mater. Tech. 122, 238-244.

[34] Lagioia, R. and Nova, R. (1995) An experimental and theoretical study of the behaviour of a calcarenite in triaxial compression. Géotechnique 45, 633-648.

[35] Lambe, T.W. and Whitman, R.V. (1969) Soil mechanics. Wiley, New York.

[36] Lewis, R.W., Jinka, A.G.K., and Gethin, D.T. (1993) Computer-aided simulation of metal powder die compaction processes. Powder Metall. Int. 25, 287-293.

[37] Lewis, R.W. and Khoei, A.R. (1998) Numerical modelling of large deformation in metal powder forming. Comput. Methods Appl. Mech. Engng. 159, 291-328.

[38] Lordi, N.G. and Cuitiño, A.M. (1997) Compaction of pharmaceuticals. MRS Bull. $22,34-37$.

[39] Maier, G. and Hueckel, T. (1979) Non-associated and coupled flow-rules of elastoplasticity for rock-like materials. Int. J. Rock Mech. Min. Sci. 16, 77-92.

[40] Oliver, J., Oller, S. and Cante, J.C. (1996) A plasticity model for simulation of industrial powder compaction processes. Int. J. Solids Struct. 33, 3161-3178.

[41] Ortiz, M. and Simo, J.C. (1986) An analysis of a new class of integration algorithms for elastoplastic constitutive relations. Int. J. Numer. Meth. Eng. 23, 353-366.

[42] Piccolroaz, A., Gajo, A. and Bigoni, D. (2002) Forming of advanced ceramics. In Selected mechanical problems in structural ceramics, Bigoni, D. Ed., pp.81-115, Polish Academy of Sciences, Warsaw.

[43] Piccolroaz, A., Bigoni, D. and Gajo, A. (2005) An elastoplastic framework for granular materials becoming cohesive through mechanical densification. Part II - elastoplastic coupling at large strain.

[44] Redanz, P. (1999) Numerical modelling of the powder compaction of a cup. Eur. J. Mech., A Solids 18, 399-413.

[45] Redanz, P. (2001) A study of stresses in powder compacted components during and after ejection. Int. J. Solids Struct. 38, 759-775.

[46] Redanz, P. and Tvergaard, V. (2003) Analysis of shear band instabilities in compaction of powders Int. J. Solids Struct. 40, 1853-1864.

[47] Reed, J.S., 1995. Principles of Ceramic Processing, Wiley \& Sons, New York.

[48] Roscoe, K.H. and Burland, J.B. (1968) On the generalized stress-strain behaviour of 'wet' clay. In Engineering Plasticity, Heyman, J. and Leckie, F.A. Eds., pp.535-609, Cambridge University Press, Cambridge.

[49] Roscoe, K.H. and Schofield, A.N. (1963) Mechanical behaviour of an idealised 'wet' clay. Proc. European Conf. on Soil Mechanics and Foundation Engineering, Wiesbaden (Essen: Deutsche Gesellshaft für Erd- und Grundbau e. V.), vol. 1, pp.47-54. 
[50] Rowe, P.W. (1962) The stress dilatancy relation for static equilibrium of an assembly of particles in contact. Proc. R. Soc. Lond. A 269, 500-527.

[51] Simo, J.C. and Hughes, T.J.R. (1987) General return mapping algorithms for rateindependent plasticity. In Constitutive laws for engineering materials: theory and applications, Desai, C.S. et al. Eds.

[52] Simo, J.C. and Ortiz, M. (1985) A unified approach to finite deformation plasticity based on the use of hyperelastic constitutive equations. Comput. Method. Appl. M. 49, 221-245.

[53] Sun, X.-K. and Kim, K.-T. (1997) Simulation of cold die compaction densification behaviour of iron and copper powders by Cam-clay model. Powder Metallurgy 40, 193-195.

[54] Thompson, R.A. (1981b) Mechanics of powder pressing: II, Finite-element analysis of end-capping in pressed green powders. Am. Ceram. Soc. Bull. 60, 244-247.

[55] Zipse, H. (1997) Finite-element simulation of die pressing and sintering of a ceramic component. J. Eur. Ceram. Soc. 17, 1707-1713.

[56] Zeuch, D.H., Grazier, J.M., Argüello, J.G. and Ewsuk, K.G. (2001) Mechanical properties and shear failure surfaces for two alumina powders in triaxial compression. J. Mater. Sci. 36, 2911-2924. 


\section{APPENDIX A. Powder characteristics}

Calibration of the model has been performed on the basis of experiments both already available and carried out on a commercial ready-to-press alumina powder ( $96 \%$ purity), manufactured by Martinswerk GmbH (Bergheim, Germany) and identified as 392 Martoxid KMS-96. The data presented by the manufacturer are given in Table 2. It can be noted from the upper part of Fig. 1 that the granules have a mean diameter of $250 \mu \mathrm{m}$.

Table 2: Granulometric and density properties of the tested alumina powder.

\begin{tabular}{||l|c||}
\hline \hline MWM 28 Vibration sieving & $3.9 \%$ \\
sieve residue $>300 \mu \mathrm{m}$ & $56.3 \%$ \\
sieve residue $>150 \mu \mathrm{m}$ & $2.5 \%$ \\
sieve residue $<63 \mu \mathrm{m}$ & 1.219 \\
\hline Bulk density $\left(\mathrm{g} / \mathrm{cm}^{3}\right)$ & 2.39 \\
\hline Green density $(\mathrm{p}=50 \mathrm{MPa})\left(\mathrm{g} / \mathrm{cm}^{3}\right)$ & 3.77 \\
\hline Fired density $\left(\mathrm{T}=1600^{\circ} \mathrm{C}, 2 \mathrm{~h}\right)\left(\mathrm{g} / \mathrm{cm}^{3}\right)$ & \\
\hline \hline
\end{tabular}




\section{APPENDIX B. Yield function gradient}

The gradient $\boldsymbol{Q}$ of the yield function (5) is [a detailed derivation can be found in (Bigoni and Piccolroaz, 2004)]

$$
\boldsymbol{Q}=\frac{\partial F}{\partial \boldsymbol{\sigma}}=a(p) \boldsymbol{I}+b(\theta) \tilde{\boldsymbol{S}}+c(\theta) \tilde{\boldsymbol{S}}^{\perp},
$$

where

$$
\tilde{\boldsymbol{S}}=\sqrt{\frac{3}{2}} \frac{\operatorname{dev} \boldsymbol{\sigma}}{q}, \quad \tilde{\boldsymbol{S}}^{\perp}=-\frac{\sqrt{2}}{\sqrt{3} q} \frac{\partial \theta}{\partial \boldsymbol{\sigma}}=\frac{1}{\sin 3 \theta}\left[\sqrt{6}\left(\tilde{\boldsymbol{S}}^{2}-\frac{1}{3} \boldsymbol{I}\right)-\cos 3 \theta \tilde{\boldsymbol{S}}\right],
$$

and

$$
\begin{aligned}
a(p) & =-\frac{1}{3} \frac{\partial f(p)}{\partial p}=\frac{M p_{c}}{3\left(p_{c}+c\right)} \frac{\left(1-m \Phi^{m-1}\right)[2(1-\alpha) \Phi+\alpha]+2(1-\alpha)\left(\Phi-\Phi^{m}\right)}{2 \sqrt{\left(\Phi-\Phi^{m}\right)[2(1-\alpha) \Phi+\alpha]}}, \\
b(\theta) & =\sqrt{\frac{3}{2}} \frac{1}{g(\theta)} \\
c(\theta) & =-\frac{\sqrt{3} \gamma \sin 3 \theta}{\sqrt{2} \sqrt{1-\gamma^{2} \cos ^{2} 3 \theta}} \sin \left[\beta \frac{\pi}{6}-\frac{1}{3} \cos ^{-1}(\gamma \cos 3 \theta)\right] .
\end{aligned}
$$

